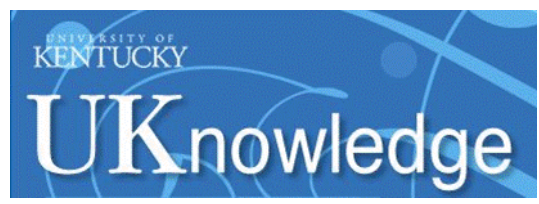

University of Kentucky

UKnowledge

$1-20-1996$

\title{
Polarization of Astronomical Maser Radiation. III. Arbitrary Zeeman Splitting and Anisotropic Pumping
}

Moshe Elitzur

University of Kentucky, moshe@pa.uky.edu

Follow this and additional works at: https://uknowledge.uky.edu/physastron_facpub

Part of the Astrophysics and Astronomy Commons, and the Physics Commons

Right click to open a feedback form in a new tab to let us know how this document benefits you.

\section{Repository Citation}

Elitzur, Moshe, "Polarization of Astronomical Maser Radiation. III. Arbitrary Zeeman Splitting and Anisotropic Pumping" (1996). Physics and Astronomy Faculty Publications. 223.

https://uknowledge.uky.edu/physastron_facpub/223

This Article is brought to you for free and open access by the Physics and Astronomy at UKnowledge. It has been accepted for inclusion in Physics and Astronomy Faculty Publications by an authorized administrator of UKnowledge. For more information, please contact UKnowledge@lsv.uky.edu. 


\section{Polarization of Astronomical Maser Radiation. III. Arbitrary Zeeman Splitting and Anisotropic Pumping}

Digital Object Identifier (DOI)

http://dx.doi.org/10.1086/176741

\section{Notes/Citation Information}

Published in The Astrophysical Journal, v. 457, no. 1, p. 415-430.

(C) 1996. The American Astronomical Society. All rights reserved.

The copyright holder has granted permission for posting the article here. 
ThE Astrophysical JoURNAL, 457:415-430, 1996 January 20

(c) 1996. The American Astronomical Society. All rights reserved. Printed in U.S.A.

\title{
POLARIZATION OF ASTRONOMICAL MASER RADIATION. III. ARBITRARY ZEEMAN SPLITTING AND ANISOTROPIC PUMPING
}

\author{
MOSHE ELITZUR \\ Department of Physics and Astronomy, University of Kentucky, Lexington, KY 40506-0055; moshe@pa.uky.edu \\ Received 1995 January 25 ; accepted 1995 July 31
}

\begin{abstract}
General solutions of the maser polarization problem are presented for arbitrary absorption coefficients. The results are used to calculate polarization for masers permeated by magnetic fields with arbitrary values of $x_{B}$, the ratio of Zeeman splitting to Doppler linewidth, and for anisotropic ( $m$-dependent) pumping. In the case of magnetic fields, one solution describes the polarization for overlapping Zeeman components, $x_{B}<1$. The $x_{B} \rightarrow 0$ limit of this solution reproduces the linear polarization derived in previous studies, which were always conducted at this unphysical limit. Terms of higher order in $x_{B}$ have a negligible effect on the magnitude of $q$. However, these terms produce some major new results. (1) The solution is realized only when the Zeeman splitting is sufficiently large that $x_{B}>\left(S_{0} / J_{s}\right)^{1 / 2}$, where $S_{0}$ is the source function and $J_{s}$ is the saturation intensity (pumping schemes typically have $S_{0} / J_{s} \sim 10^{-5}$ to $10^{-8}$ ). When this condition is met, the linear polarization requires $J / J_{s} \gtrsim x_{B}$, where $J$ is the angle-averaged intensity. This condition generally requires considerable amplification, but is met long before saturation $\left(J / J_{s} \geq 1\right)$. (2) The linear polarization is accompanied by circular polarization, proportional to $x_{B}$. Because $x_{B}$ is proportional to the transition wavelength, the circular polarization of $\mathrm{SiO}$ masers should decrease with rotation quantum number, as observed. In the absence of theory for $x_{B}<1$, previous estimates of magnetic fields from detected maser circular polarization had to rely on conjectures in this case and generally need to be revised downward. The fields in SiO masers are $\sim 2-10 \mathrm{G}$ and were overestimated by a factor of 8 . The $\mathrm{OH}$ maser regions around supergiants have fields of $\sim 0.1-0.5$ $\mathrm{mG}$, which were overestimated by factors of 10-100. The fields were properly estimated for OH/IR masers ( $\lesssim 0.1 \mathrm{mG})$ and $\mathrm{H}_{2} \mathrm{O}$ masers in star-forming regions $(\sim 15-50 \mathrm{mG})$. (3) Spurious solutions that required stability analysis for their removal in all previous studies are never reproduced here; in particular, there are no stationary physical solutions for propagation at $\sin ^{2} \theta<\frac{1}{3}$, where $\theta$ is the angle from the direction of the magnetic field, so such radiation is unpolarized. These spurious solutions can be identified as the $x_{B}=0$ limits of nonphysical solutions and they never arise a finite values of $x_{B}$, however small. (4) Allowed values of $\theta$ are limited by bounds that depend both on Zeeman splitting and frequency shift from line center. At $x_{B} \lesssim 10^{-3}$, the allowed phase space region encompasses essentially all frequencies and $\sin ^{2} \theta>\frac{1}{3}$. As the field strength increases, the allowed angular region shrinks at a frequency-dependent rate, leading to contraction of the allowed spectral region. This can result in narrow maser features with linewidths smaller than the Doppler width and substantial circular polarization in sources with $x_{B} \gtrsim 0.1$. When $x_{B} \geq 0.7$, all frequencies and directions are prohibited for the stationary solution and the radiation is unpolarized.

Another solution describes the polarization when the Zeeman components separate. This occurs at line center when $x_{B}>1$ and at one Doppler width when $x_{B}>2$. The solution is identical to that previously identified in the $x_{B} \rightarrow \infty$ limit, and applies to $\mathrm{OH}$ masers around $\mathrm{H}$ II regions. A significant new result involves the substantial differences between the $\pi$ - and $\sigma$-components for most propagation directions, differences that persist into the saturated domain. Overall, $\mathrm{H} \mathrm{II} / \mathrm{OH}$ regions should display a preponderance of $\sigma$-components. The absence of any $\pi$-components in $\mathrm{W} 3(\mathrm{OH})$ finds a simple explanation as maser action in a magnetic field aligned within $\sin ^{2} \theta<\frac{2}{3}$ to the line of sight.
\end{abstract}

Subject headings: atomic processes - magnetic fields - masers - polarization — radiative transfer

\section{INTRODUCTION}

Maser radiation is frequently polarized. Polarization generation is usually attributed to the presence of a magnetic field, which plays a dual role in this process. First, the magnetic field introduces a quantization axis, enabling a meaningful distinction between magnetic quantum numbers. This occurs when the field is sufficiently strong that the gyrorate exceeds all other relevant microscopic rates. Once this condition is met, which is virtually always the case in astronomical masers, further increases in field strength are irrelevant; as far as this aspect of the problem is concerned, the field strength can be ignored altogether.

Second, the magnetic field shifts the energies of magnetic sublevels by the Zeeman effect, splitting each line into com- ponents with different $\Delta m$. The relevant dimensionless parameter for the significance of the Zeeman splitting $\Delta v_{B}$ is its ratio to the Doppler linewidth $\Delta v_{\mathrm{D}}$,

$$
x_{B}=\frac{\Delta v_{B}}{\Delta v_{\mathrm{D}}}=14 g \lambda \frac{B}{\Delta v_{5}} .
$$

In the last equality $g$ is the Lande factor with respect to the Bohr magneton, $\lambda$ is the transition wavelength in $\mathrm{cm}, B$ is the field strength in gauss and $\Delta v_{5}$ is the Doppler width in $\mathrm{km} \mathrm{s}^{-1}$. Because the Zeeman shifts are proportional to the field strength, the absorption coefficients for the various Zeeman components vary with $x_{B}$; thus this aspect of the problem can be expected to introduce a direct dependence of the polarization on $x_{B}$. 
A general theory for the dependence of maser polarization on $x_{B}$ has not been formulated thus far. Instead, ever since the seminal work by Goldreich, Keeley, \& Kwan (1973, GKK hereafter) all maser polarization studies, including the first two in this series (Elitzur 1991, 1993; Papers I and II hereafter), were performed in one of two limits - either $x_{B} \rightarrow \infty$ or $x_{B} \rightarrow 0$. Absorption coefficients were derived from rate equations in which these limits were assumed beforehand, not by taking the appropriate limits of general expressions for arbitrary $x_{B}$. This procedure seems adequate when the Zeeman components are fully separated because the absorption coefficients drop exponentially in the line wings. Once $x_{B}$ greatly exceeds unity, each component can be treated as an independent line, the dependence on $x_{B}$ can be expected to be insignificant and $x_{B} \rightarrow \infty$ is a proper, physical limit.

The case of overlapping Zeeman components, $x_{B} \ll 1$, is considerably more problematic. This case was always treated in the limit $x_{B}=0$, which is of course unphysical-in the absence of a magnetic field there is no quantization axis and no polarization. In essence, current theory is predicated on the implicit assumption that, although $x_{B}$ is finite, it is sufficiently small that $x_{B}=0$ can be used, but only in the expressions for the absorption coefficients (which are then derived from rate equations that assume this limit at the outset). It should be noted that, although unphysical, the $x_{B} \rightarrow 0$ limit of a complete theory can still be legitimately explored; the actual polarization varies with $x_{B}$ in some fashion and may well contain $x_{B^{-}}$ independent terms that provide finite values in that limit. But such a limit can be considered only within the context of a general theory in which the $x_{B}$ variation is shown explicitly. The deficiency of current theory is that this variation is completely unknown because the limit $x_{B}=0$ is assumed at the outset in the calculation of the absorption coefficients, making it an uncontrolled approximation. It is impossible to assess how small $x_{B}$ must be for any results to be valid and whether any of them may actually reflect singularities that arise only at $x_{B}=0$.

All the theoretical tools for development of a general theory for maser polarization are available by now. Litvak (1975) has formulated the general theory for transport of line radiation and Paper II has clarified the procedures introduced in GKK for identifying maser stationary polarizations. These ingredients are combined in $\S 2$ to produce the general solutions for maser polarization for arbitrary absorption coefficients. The subsequent two sections utilize these results for masers in magnetic fields $-\$ 3$ presents the solution when the Zeeman components separate, $\S 4$ when they overlap. Section 5 presents the general solution for polarization in sources where the pumping is $m$-dependent with an arbitrary degree of anisotropy. All the solutions are tabulated for handy reference and the results are summarized and discussed in $\S 6$. This final section also contains comparison with observations and can be read on its own, independent of the rest of the paper.

\section{POLARIZATION SOLUTIONS}

A full description of the electromagnetic radiation field involves the 4-vector of its Stokes parameters $S=(I, Q, U, V)$. The general transfer equation for $\boldsymbol{S}$ in the case of line radiation was derived by Litvak (1975) and is listed in Appendix A. When the source terms can be neglected, which is the case for maser radiation, the radiative transfer equation is

$$
\frac{d \boldsymbol{S}}{d l}=\boldsymbol{R} \cdot \boldsymbol{S},
$$

where the matrix $\boldsymbol{R}$ can be read off equation (A3). Neglect of the source terms, further discussed in $\S 4.2 .1$, is justified in essentially all cases of interest. Typically, the brightness temperature of an astronomical maser exceeds its excitation temperature by at least eight orders of magnitude, so only the properties of the self-amplified terms are relevant.

\subsection{Stationary Polarizations}

The polarization is characterized by $\Pi=(q, u, v)$, the 3 vector of normalized Stokes parameters $q=Q / I, u=U / I$, $v=V / I$ (see Fig. 1). Configurations that obey $d \Pi / d l=0$ have stationary polarization that does not vary with propagation and can be expected to describe observed maser radiation; all other polarizations will evolve until they lock into a stationary state. The condition of stationary polarization is

$$
q^{\prime}=u^{\prime}=v^{\prime}=0
$$

where a prime denotes derivative along the path. Because $q=Q / I, q^{\prime}=0$ is equivalent to $Q^{\prime} / Q=I^{\prime} / I$, with a similar relation for $U$ and $V$. Therefore, stationary polarizations obey

$$
\frac{I^{\prime}}{I}=\frac{Q^{\prime}}{Q}=\frac{U^{\prime}}{U}=\frac{V^{\prime}}{V} \equiv \lambda,
$$

where $\lambda$ is some (unknown) factor, common to all four Stokes parameters. So stationary polarizations obey $d S / d l=\lambda S$ and comparison with the radiative transfer equation shows that they are the eigenvectors of the matrix $\boldsymbol{R}$, with $\lambda$ the corresponding eigenvalues. All four Stokes parameters of a stationary polarization state obey an equation identical to that for the intensity of a scalar maser, the widely used model that treats the radiation electric field as a scalar, with $\lambda$ its (single) absorption coefficient. With minor changes, the extensive theory derived for scalar masers can be carried over to polarized masers. In particular, the Stokes parameters of stationary solutions grow as $\exp \left(\int \lambda d l\right)$.

\subsubsection{The Solutions}

Because physical solutions must have $I \neq 0$, the eigenvalue equations, obtained from equation (A3), can be divided by $I$ to produce a set of equations for the normalized Stokes

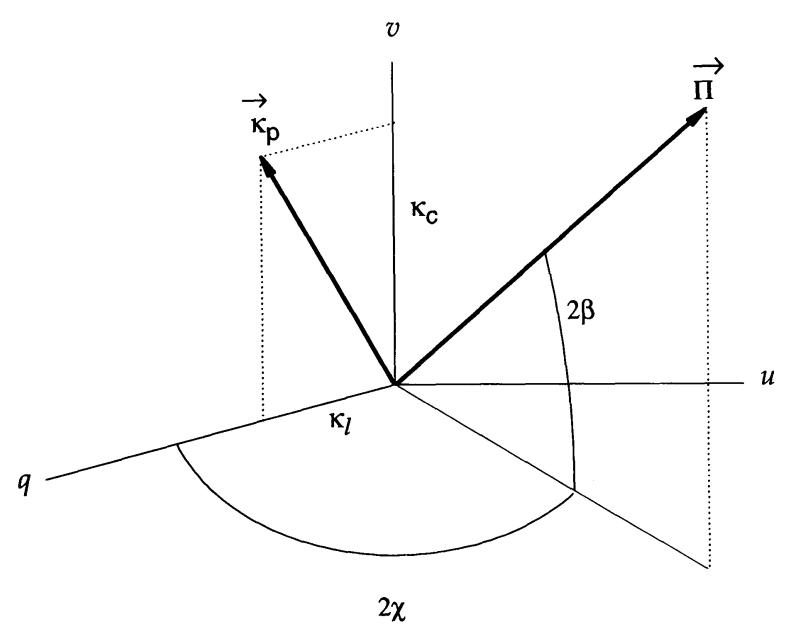

Fig. 1.-The polarization vector $\Pi$ and the vector $\boldsymbol{\kappa}_{p}$ that controls its radiative transfer in the space defined by the normalized Stokes parameters $q$, $u$, and $v$. 
parameters:

$$
\begin{aligned}
\left(\lambda-\kappa_{m}\right) & =\kappa_{l} q+\kappa_{c} v, \\
\left(\lambda-\kappa_{m}\right) q & =\kappa_{l}, \\
\left(\lambda-\kappa_{m}\right) u & =0, \\
\left(\lambda-\kappa_{m}\right) v & =\kappa_{c} .
\end{aligned}
$$

Now multiply equation (2.4b) by $\kappa_{l}$, equation (2.4d) by $\kappa_{c}$ and combine. Inserting $\kappa_{l} q+\kappa_{c} v$ from equation (2.4a) leads to

$$
\left(\lambda-\kappa_{m}\right)^{2}=\kappa_{l}^{2}+\kappa_{c}^{2} \equiv \kappa_{p}^{2},
$$

so the eigenvalues are $\lambda=\kappa_{m} \pm \kappa_{p}$. When $\kappa_{p} \neq 0$, the solution is

$$
\lambda=\kappa_{m}+\kappa_{p}, \quad q=\frac{\kappa_{l}}{\kappa_{p}}, \quad u=0, \quad v=\frac{\kappa_{c}}{\kappa_{p}},
$$

which will be called a Type 1 Solution. Because $\kappa_{p}>0$, the solution with $\lambda=\kappa_{m}-\kappa_{p}$ has a lower growth rate and can be dismissed. ${ }^{1}$ Since $q^{2}+v^{2}=1$, type 1 solutions describe fully polarized radiation. Note that although we obtained explicit expressions for the fractional polarizations, $\kappa_{l}$ and $\kappa_{c}$ themselves can depend on $q$ and $v$. In that case the polarization may not be fully specified, with the solution only providing a selfconsistency relation for $q$ and $v$.

When $\kappa_{p}=0$ (i.e., both $\kappa_{l}$ and $\kappa_{c}$ vanish), $\lambda=\kappa_{m}$. All the eigenvalue equations are obeyed trivially and the polarization is not constrained by the eigenvalue problem. However, only certain polarization configurations can maintain $\kappa_{l}=\kappa_{c}=0$, if at all, so the polarization is determined in this case from the conditions imposed by the vanishing of the off-diagonal elements of the matrix $\boldsymbol{R}$. That is, the solution is

$$
\lambda=\kappa_{m}, \quad \kappa_{l}=\kappa_{c}=0,
$$

which will be called a Type 0 Solution. Just as in the general case of type 1 polarization, the Stokes parameters are determined implicitly. Because the off-diagonal $\kappa_{l}$ and $\kappa_{c}$ determine all the possible differences among the three basic absorption coefficients $\kappa^{\Delta m}$, their vanishing implies

$$
\kappa^{+}=\kappa^{-}=\kappa^{0}=\kappa_{m}=\lambda .
$$

Type 0 polarization is obtained when the absorption coefficients of all three $\Delta m$ transitions are equal to each other. In the case of $J=1 \rightarrow 0$ transitions, discussed in Appendix A, this implies equal populations for all the magnetic substates of the upper level, a point first noted in Paper I. Obviously, such a situation is impossible when only one $\Delta m$ transition is involved, as is the case for fully developed Zeeman patterns. Type 0 solutions, which can be identified using either of the last two equations, are possible only when the Zeeman components overlap. Note that such a solution is not the $\kappa_{p} \rightarrow 0$ limit of a type 1 polarization; although the eigenvalue is properly reproduced, $q$ and $v$ are indeterminate in that limit.

\subsection{Miscellaneous Properties of the Solutions}

The polarization solutions can be further understood with the aid of

$$
\boldsymbol{\kappa}_{p}=\left(\kappa_{l}, 0, \kappa_{c}\right)
$$

\footnotetext{
${ }^{1}$ All the explicit type 1 polarizations derived below have $\kappa_{p}=\kappa_{m}$ and this choice of sign gives a solution that does not grow at all.
}

a vector in $(q, u, v)$-space whose magnitude is $\left|\boldsymbol{\kappa}_{p}\right|=\kappa_{p}$ (see Fig. 1 ; this vector was denoted $\boldsymbol{\beta}$ in Litvak's paper); note that $\boldsymbol{\kappa}_{p} \cdot \boldsymbol{\Pi}=\kappa_{l} q+\kappa_{c} v$. With this vector, the radiative transfer equations can be written as

$$
\begin{aligned}
& \frac{d I}{d l}=\left(\kappa_{m}+\kappa_{p} \cdot \Pi\right) I, \\
& \frac{d \Pi}{d l}=\kappa_{p}-\left(\kappa_{p} \cdot \Pi\right) \Pi=\left[\Pi \times \kappa_{p}\right] \times \Pi+\left(1-\Pi^{2}\right) \kappa_{p} .
\end{aligned}
$$

The polarization is stationary $\left(\boldsymbol{\Pi}^{\prime}=0\right)$ when $\boldsymbol{\kappa}_{p}$ vanishes, corresponding to a type 0 solution. When $\kappa_{p} \neq 0$, the polarization is stationary only when $\Pi$ is along $\boldsymbol{\kappa}_{p}$ and has a magnitude of unity (full polarization), corresponding to a type 1 solution. This result has a simple interpretation in terms of the individual polarization modes $\boldsymbol{\Pi}_{n}$, which make up the overall polarization through the ensemble average $\boldsymbol{\Pi}=\left\langle\boldsymbol{\Pi}_{n}\right\rangle$ (see Paper II). Because $\left|\boldsymbol{\Pi}_{n}\right|^{2}=1$, the radiative transfer of individual modes involves pure rotation with the angular velocity $\Pi_{n} \times \kappa_{p}$, which is different for different modes. The rotation comes to a halt only when $\boldsymbol{\Pi}_{n}$ overlaps $\boldsymbol{\kappa}_{p}$. The overall polarization $\boldsymbol{\Pi}$ settles into the stationary solution when all individual modes have rotated into overlap with $\boldsymbol{\kappa}_{p}$, producing a fully polarized radiation field whose polarization is determined by $\kappa_{l}: \kappa_{c}$, as is evident from Figure 1.

With this form, the radiative transfer equations admit the formal solution

$$
I=I_{0} e^{\tau_{m}+\tau_{\pi}}, \quad \Pi^{2}=1-\left(1-\boldsymbol{\Pi}_{0}^{2}\right) e^{-2 \tau_{\pi}},
$$

where

$$
\tau_{m}=\int_{l_{0}}^{l} \kappa_{m} d l, \quad \tau_{\pi}=\int_{l_{0}}^{l} \boldsymbol{\Pi} \cdot \boldsymbol{\kappa}_{p} d l
$$

and where $I_{0}$ and $\Pi_{0}$ are the intensity and polarization at some fiducial point $l_{0}$ along the path. The vanishing of $\kappa_{p}$ produces $\tau_{\pi}=0$ and stationary polarization whose intensity increases exponentially with $\tau_{m}$, i.e., a type 0 solution (eq. [2.7]). In addition, full polarization always maintains a fixed magnitude. It maintains also a fixed direction, becoming stationary, when $\boldsymbol{\Pi}$ overlaps $\kappa_{p}$, leading to $\tau_{\pi}=\int \kappa_{p} d l$ and the type 1 solution of equation (2.6).

\subsubsection{Scalar versus Polarized Masers}

The general formal solution of the radiative transfer problem helps illustrate a fundamental difference, first noted in Elitzur (1995), between the radiation fields of scalar and polarized masers. In the scalar maser model, different radiative modes correspond to different initial intensities $I_{0}$ and the growth of each mode follows the formal solution

$$
I=I_{0} e^{\tau},
$$

where $\tau=\int \kappa d l$ and $\kappa$ is the (only) absorption coefficient, the equivalent of $\kappa_{m}$. In the unsaturated regime $\kappa$ is a constant independent of intensity, and the growth is exponential with path length. Therefore, the scalar unsaturated maser is a purely linear amplifier; its growth rate is independent of intensity, i.e., it is mode-invariant. All the modes grow at the same rate and the mode distribution is unaffected by the amplification. This is the reason why the need to consider ensemble averages usually does not even arise in the scalar maser model.

The inclusion of polarization changes the situation fundamentally. The formal solution for $I$ is now given in equation 
(2.11) and similar expressions can be listed for all other Stokes parameters. As with the scalar maser, when radiative interactions are negligible in the unsaturated domain the absorption coefficients are constant and their integrals increase linearly with path length, resulting in exponential amplification. However, even though the absorption coefficients are independent of the Stokes parameters in this case, the optical depth $\tau_{\pi}$ still does depend on them. Because of the explicit dependence of $\tau_{\pi}$ on $\boldsymbol{\Pi}$, the growth of the Stokes parameters depends on the Stokes parameters themselves, in spite of the fact that the population inversions are unaffected by radiative interactions. In order to get a quantity whose growth is modeinvariant, similar to the intensity of a scalar maser, the offdiagonal optical depth $\tau_{\pi}$ must be eliminated. This is easily accomplished by combining the expressions for $I$ and $\Pi^{2}$ in equation (2.11) to produce

$$
I\left(1-\Pi^{2}\right)^{1 / 2}=I_{0}\left(1-\Pi_{0}^{2}\right)^{1 / 2} e^{\tau_{m}},
$$

the analog of equation (2.13) for scalar masers. In the unsaturated domain, $\tau_{m}$ again is the same for all modes. However, now the invariant growth does not apply to any single Stokes parameter; instead it applies to $I^{2}\left(1-\Pi^{2}\right)=$ $I^{2}-Q^{2}-U^{2}-V^{2}$, a quadratic form involving all four Stokes parameters. ${ }^{2}$

Since the growth rates of the Stokes parameters depend on the Stokes parameters themselves, the unsaturated polarized maser is a nonlinear amplifier. As a result, a calculation of the evolution of ensemble-averaged Stokes parameters, rather than merely deriving their stationary values as done here, invariably requires numerical simulations that properly evolve an ensemble of waves. Such a simulation, frequently performed in plasma studies, will not be attempted here. It would be similar to a demonstration of the approach to Maxwellian of a particle velocity distribution or the approach to Planckian of a photon distribution. While such demonstrations are valuable, they are considerably more difficult than the mere derivation of either equilibrium distribution, the equivalent of the stationary solution of the maser polarization problem derived here.

Stationary polarization requires that the radiation maintain the same normalized Stokes parameters during propagation through the source, even as the Stokes parameters themselves grow by maser amplification. However, the solutions for such polarizations are determined by the vector $\boldsymbol{\kappa}_{p}$, and in general $\boldsymbol{\kappa}_{p}$ can be expected to vary both in magnitude and direction, reflecting variations of the level populations as a result of interaction with the growing maser radiation. A solution is not guaranteed; indeed, stationary polarization may be altogether impossible in any particular region of phase space, in which case the radiation is unpolarized. We proceed now to derive the stationary polarizations, wherever they exist, for various magnetic field strengths.

\section{FULLY RESOLVED ZEEMAN PATTERN}

When the Zeeman pattern is fully developed, only one absorption coefficient is different from zero at the frequency of each component. Thus the condition $\kappa^{+}=\kappa^{-}=\kappa^{0}$ cannot be fulfilled and type 0 polarization is impossible; only type 1

\footnotetext{
${ }^{2}$ This result can also be obtained directiy from the radiative transfer equation (A3) which leads to

$$
\frac{d}{d l}\left(I^{2}-Q^{2}-U^{2}-V^{2}\right)=2 \kappa_{m}\left(I^{2}-Q^{2}-U^{2}-V^{2}\right)
$$
}

polarization can grow in this case. In addition, the direction of the vector $\boldsymbol{\kappa}_{p}$ (though not its magnitude) is constant, unaffected by the variation of the level populations with saturation.

At the frequency of a $\pi$-component, only $\kappa^{0}$ does not vanish. Therefore, $\kappa_{c}=0$ while $\kappa_{m}=\kappa_{p}=-\kappa_{l}=\frac{1}{2} \kappa^{0} \sin ^{2} \theta$. From equation (2.6), the polarization solution is

$$
\kappa^{\pi}=\kappa^{0} \sin ^{2} \theta, \quad q=-1, \quad v=0,
$$

where $\kappa^{\pi}\left(=\kappa_{m}+\kappa_{p}\right)$ is the eigenvalue, i.e., the maser growth rate. In the case of $\sigma$-components only $\kappa^{+}\left(\kappa^{-}\right)$does not vanish, so $\kappa_{l}=\frac{1}{4} \kappa^{ \pm} \sin ^{2} \theta, \quad \kappa_{c}= \pm \frac{1}{2} \kappa^{ \pm} \cos \theta, \quad$ and $\kappa_{m}=\kappa_{p}=$ $\frac{1}{4} \kappa^{ \pm}\left(1+\cos ^{2} \theta\right)$. From equation (2.6), the polarization solution is now

$$
\begin{aligned}
\kappa^{\sigma} & =\frac{1}{2} \kappa^{ \pm}\left(1+\cos ^{2} \theta\right), \quad q=\frac{\sin ^{2} \theta}{1+\cos ^{2} \theta}, \\
v & = \pm \frac{2 \cos \theta}{1+\cos ^{2} \theta},
\end{aligned}
$$

where $\kappa^{\sigma}$ is the appropriate growth rate. These polarizations are identical to those derived in GKK for fully resolved Zeeman patterns. Since the detailed expressions for the absorption coefficients $\kappa^{\Delta m}$ were not even specified, these solutions apply to all spins and degrees of saturation, in agreement with the conclusions of Paper $I$.

Explicit expressions for $\kappa^{\pi}$ and $\kappa^{\sigma}$ can be derived only in the context of a specific maser model. Appendix A provides the general solution for the absorption coefficients $\kappa^{\Delta m}$ of a spin $1 \rightarrow 0$ transition (eq. [A7]). When the Zeeman components are fully separated, ground state particles with a given velocity interact only with radiation shifted by the same amount from each of the three line centers; for example, particles with zero velocity interact only with the line center radiation of each Zeeman component. Therefore, each $J_{\Delta m}$ and $\kappa_{0}^{\Delta m}$ in equation (A7) must be considered at the same frequency shift from the line center of the $(1, m) \rightarrow 0$ transition. If pumping of the upper levels is $m$-independent, i.e., $P_{1 m}=P_{1}$, then $\kappa_{0}^{\Delta m}=\kappa_{0} \propto$ $\left(P_{1}-P_{0}\right) / \Gamma$ and the absorption coefficients are

$$
\kappa^{\Delta m}=\frac{\kappa_{0}}{1+f_{\Delta m} J_{\Delta m} / J_{s}} E,
$$

where

$$
E=\left(1+\sum_{\Delta m} e_{\Delta m}\right)^{-1}
$$

The common factor $E$ varies from 1 when all three components are unsaturated $\left(e_{\Delta m}=0\right)$ to $\frac{1}{4}$ when all are saturated $\left(e_{\Delta m}=1\right)$. Inserting the polarization from the appropriate solution [eqs. (3.1) and (3.2)], the absorption coefficients of the Zeeman components become

$$
\begin{aligned}
\kappa^{\pi} & =\frac{\kappa_{0} \sin ^{2} \theta}{1+\sin ^{2} \theta J_{\pi} / J_{s}} E, \\
\kappa^{\sigma} & =\frac{\kappa_{0}\left(1+\cos ^{2} \theta\right) / 2}{1+(1 / 2)\left(1+\cos ^{2} \theta\right) J_{\sigma} / J_{s}} E,
\end{aligned}
$$

where $J_{\pi}$ and $J_{\sigma}$ are the corresponding angle-averaged intensities. These results provide reasonable approximations for the absorption coefficients at frequency $x$ when $x_{B}>1+x$ because the matching spectral segments of neighboring lines are then separated by at least a full Doppler width. Therefore, 
these results apply at each line center when $x_{B}>1$, and as $x_{B}$ increases their applicability region spreads to additional frequencies, further removed from line center. When $x_{B} \gtrsim 2$, these absorption coefficients should describe adequately the entire portion of interest of each line.

Apart from the factor $E$, for propagation along the axis the absorption coefficients of the $\sigma$-components are identical to those of a scalar maser with the same pumping scheme. The same applies to the $\pi$-component, but for propagation perpendicular to the axis. Moving to other directions, in each case the unsaturated absorption coefficient is reduced by an angledependent factor. When both components are unsaturated, their intensities grow according to

$$
\begin{aligned}
& I_{\pi}=S_{0} \times \exp \left(\kappa_{0} l \sin ^{2} \theta\right), \\
& I_{\sigma}=S_{0} \exp \left(\frac{1}{2} \kappa_{0} l\right) \times \exp \left(\frac{1}{2} \kappa_{0} l \cos ^{2} \theta\right),
\end{aligned}
$$

where $S_{0}$ is the source function. These angular distributions are highly anisotropic; with $\kappa_{0} l=10$, corresponding to unsaturated maser operation in typical pumping schemes, $I_{\sigma}$ drops to $1 / e$ of its peak at $\theta=27^{\circ}$ and the same holds for $I_{\pi}$ at $90^{\circ}-\theta=18^{\circ}$. The anisotropy introduces a high degree of asymmetry between the $\pi$ - and $\sigma$-components, which have equal intensities for the same growth length at $\sin ^{2} \theta=\frac{2}{3}$, i.e., $\theta=55^{\circ}$. At $0 \leq \theta<55^{\circ}$ the $\sigma$-components have a higher intensity, at $55^{\circ}<\theta \leq 90^{\circ}$ the $\pi$-component is stronger. The regions where each Zeeman component dominates have comparable sizes, but the enhancement of the $\sigma$-components in their region of dominance is more prominent. Moving away from $\theta=55^{\circ}$, the ratio $\kappa^{\pi} / \kappa^{\sigma}$ reaches a maximum of 2 for propagation at $\theta=90^{\circ}$ while the inverse ratio $\kappa^{\sigma} / \kappa^{\pi}$ becomes 2 already at $\theta=39^{\circ}$, increasing further without bound as the magnetic axis is approached. Therefore, an overall preponderance of $\sigma$ components can be expected among all unsaturated maser sources with comparable physical conditions.

When both components saturate, the $\theta$-dependence of their absorption coefficients disappears and they both obey $\kappa J=$ $\frac{1}{4} \kappa_{0} J_{s} \propto P_{1}-P_{0}$. Saturated regions have equal production rates of $\sigma$ - and $\pi$-photons independent of inclination to the magnetic axis. However, in most cases the lengths of such regions will be $\theta$-dependent and different for the two components. For each transition, the unsaturated absorption coefficient is reduced by a $\theta$-dependent factor and the corresponding saturation intensity is increased by the same factor. Both effects cause the optical depth that brings saturation to increase, lengthening the unsaturated region. If $\tau_{s}$ denotes the optical depth required for the saturation of a scalar maser in the linear geometry, the corresponding saturation optical depths of the Zeeman components are

$$
\begin{aligned}
\tau_{s}^{\pi} & =\frac{\tau_{s}-\ln \sin ^{2} \theta}{\sin ^{2} \theta}, \\
\tau_{s}^{\sigma} & =\frac{\tau_{s}-\ln (1 / 2)\left(1+\cos ^{2} \theta\right)}{(1 / 2)\left(1+\cos ^{2} \theta\right)} .
\end{aligned}
$$

These results follow from equation (3.4) and general expressions for linear masers (Elitzur 1990). They show that saturation, and $\theta$-independent maser production, generally requires path length longer than $\tau_{s}$. But whereas the saturation optical depth of $\sigma$-components can only be as large as $\sim 2 \tau_{s}$, for the $\pi$-component it can become much larger, diverging when $\theta \rightarrow 0$ (reflecting the fact that this component does not grow for propagation along the axis). Once the maser length in a given direction exceeds that required for saturation of the weak component, the intensities of both components continue to grow at the common rate $\frac{1}{4} \kappa_{0} J_{s}$ per unit length, maintaining a constant difference

$$
J_{\sigma}-J_{\pi}=\frac{1}{4} J_{s}\left(\tau_{s}^{\pi}-\tau_{s}^{\sigma}\right)
$$

The indirect coupling through the common factor $E$ (eq. [3.3]) introduces an additional element of asymmetry into the maser growth pattern. In a region where one Zeeman component is saturated but the other is not, $E$ is smaller than its unsaturated limit of 1 but larger than its saturated limit of $\frac{1}{4}$. Therefore, the growth rate of the stronger, saturated component is larger than what it would be were all components saturated, at the expense of the weaker component whose unsaturated growth rate is suppressed. When only the $\sigma$ components are saturated, $\kappa_{0}^{\pi}$ is reduced by a factor of 3 , and when only the $\pi$-component is saturated, $\kappa_{0}^{\sigma}$ is reduced by a factor of 2. This suppression of the weak Zeeman component by the strong one should add to the dominance of $\sigma$ components in maser sources with $x_{B}>1$.

\section{OVERLAPPING ZEEMAN COMPONENTS}

When $x_{B}<1$, the overlap of Zeeman components engulfs most of the line cores. In the maser model discussed in Appendix A, ground state particles at a given velocity can interact with different Zeeman components at the same frequency so all quantities in equations (A7) for the absorption coefficients must now be considered at a given frequency; in particular, $J_{\Delta m}=J$, the angle-averaged intensity at that frequency. Because of the Zeeman splitting, a given frequency corresponds to different shifts from the line centers of the three Zeeman components. The $(1, m) \rightarrow 0$ transition is centered on the frequency $v_{m}=v_{0}-m \Delta v_{B}$ and the argument of the corresponding Doppler profile is $\left(v-v_{m}\right) / \Delta v_{D}=x+m x_{B}$, where $x=\left(v-v_{0}\right) / \Delta v_{D}$ is the dimensionless frequency shift from the center of the unperturbed line. Assuming $m$-independent pumping, the unsaturated absorption coefficients of the three transitions are equal at their respective line centers, $\kappa_{0}^{\Delta m}\left(v_{m}\right)=$ $\kappa_{0} \propto\left(P_{1}-P_{0}\right) / \Gamma$, so

$$
\kappa_{0}^{\Delta m}(x)=\kappa_{0} \exp \left[-\left(x+x_{B} \Delta m\right)^{2}\right] .
$$

In spite of the $m$-independent pumping, the ratios of absorption coefficients vary across the line because of the Zeeman shifts. These variations are conveniently expressed in terms of the ratios

$$
\begin{aligned}
& R_{1}=\frac{\kappa_{0}^{+}+\kappa_{0}^{-}}{2 \kappa_{0}^{0}}=e^{-x_{B}{ }^{2}} \cosh \left(2 x x_{B}\right) \simeq 1-x_{B}^{2}\left(1-2 x^{2}\right), \\
& R_{c}=\frac{\kappa_{0}^{+}-\kappa_{0}^{-}}{2 \kappa_{0}^{0}}=-e^{-x_{B}^{2}} \sinh \left(2 x x_{B}\right) \simeq-2 x x_{B} .
\end{aligned}
$$

In each case, the second equality holds for any $x_{B}$ while the last relation provides the appropriate small- $x_{B}$ limit, a result valid to second order in $x_{B}$ and all frequency shifts with $2 x x_{B}<1$. The constraints imposed on $\kappa_{0}^{\Delta m}$ by the inequalities of equation (A12) produce $\left|1-R_{1}\right|<\frac{1}{2}$, or

$$
x_{B}^{2}<\frac{1}{2\left|1-2 x^{2}\right|} .
$$

At any frequency $x$, the Zeeman splitting must obey this bound to ensure that all three transitions remain inverted when they are saturated. In particular, fulfillment of this constraint over 
the entire central portion of the line, $|x| \leq 1$, restricts the Zeeman splitting to $x_{B}<1 / 2^{1 / 2}$.

When the three unsaturated absorption coefficients are expressed in terms of $\kappa_{0}^{0}$ and the ratios $R_{1}$ and $R_{c}$, the explicit results for the absorption coefficients (eq. [A9]) become $\kappa^{\Delta m}=$ $\kappa_{0}^{0} K^{\Delta m} / D$, where $K^{\Delta m}$ are dimensionless quadratic forms in $J / J_{s}$. With these expressions, $\kappa_{m}, \kappa_{l}$, and $\kappa_{c}$ take similar forms whose numerators are

$$
\begin{aligned}
K_{m}= & \frac{1}{2} \llbracket \sin ^{2} \theta+R_{1}\left(1+\cos ^{2} \theta\right) \\
& +\left(J / J_{s}\right)\left\{\left(1+\cos ^{2} \theta\right)\left[f_{1}\left(2-3 R_{1}\right)+2 R_{1}-1\right]\right. \\
& \left.+2 \sin ^{2} \theta f_{1}\left(2-R_{1}\right)-w R_{c}\left(5+\cos ^{2} \theta\right)\right\} \\
& +\left(J / J_{s}\right)^{2}\left\{\left(1+\cos ^{2} \theta\right) f_{0}\left[f_{1}\left(2 R_{1}-1\right)-4 w R_{c}\right]\right. \\
& \left.+\sin ^{2} \theta\left(2 R_{1}-3\right)\left(w^{2}-f_{1}^{2} \sin ^{2} \theta\right)\right\} \rrbracket \\
K_{l}= & \frac{1}{2} \sin ^{2} \theta \llbracket\left[R_{1}-1\right. \\
& +\left(J / J_{s}\right)\left[2 R_{1}-1-f_{1}\left(R_{1}+2\right)-w R_{c}\right] \\
& +\left(J / J_{s}\right)^{2}\left\{f_{0}\left[f_{1}\left(2 R_{1}-1\right)-4 w R_{c}\right]\right. \\
& \left.\quad-\left(f_{1}^{2}-w^{2}\right)\left(3-2 R_{1}\right)\right\} \rrbracket \\
K_{c}= & \cos \theta\left\{R_{c}+\left(J / J_{s}\right)\left[R_{c}\left(2-f_{1}\right)-w R_{1}\right]\right. \\
& \left.+\left(J / J_{s}\right)^{2} f_{0}\left[4 f_{1} R_{c}-w\left(2 R_{1}-1\right)\right]\right\} .
\end{aligned}
$$

In each of these relations the $J$-independent term provides the $J=0$ (unsaturated) limit of the corresponding absorption coefficient (since the $J$-independent term in $D$ is 1 ). There is a fundamental difference between these terms for the diagonal and off-diagonal absorption coefficients when $x_{B}<1$. While $K_{m}(J=0) \simeq 1$ in this case, $\left|K_{l}(0)\right|$ and $\left|K_{c}(0)\right|$ are proportional to $\left|R_{1}-1\right| \sim x_{B}^{2}$ and $\left|R_{c}\right| \sim x_{B}$, respectively, and thus are much smaller than unity. As a result, although the $J$ independent term provides an adequate approximation for $\kappa_{m}$ in the entire unsaturated domain $\left(J / J_{s}<1\right), \kappa_{l}$ and $\kappa_{c}$ are dominated by $J$-dependent terms long before saturation $\left(J / J_{s}=1\right)$; that is, the dependence on $J$ of the off-diagonal absorption coefficients becomes significant at intensities well inside the unsaturated domain, either $J / J_{s} \sim x_{B}^{2}$ or $J / J_{s} \sim x_{B}$. The reason for this difference is simple. While the $J$ dependence of $\kappa_{m}$ reflects only the difference between the mean populations of the upper and lower levels, the corresponding dependence of $\kappa_{l}$ and $\kappa_{c}$ reflects also population differences among the magnetic substates of the upper level. And those are affected by radiative interactions long before saturation reduces the overall population inversion.

\subsection{Type 1 Polarization}

Type 1 solutions can be derived from the equation $q K_{c}\left(q, v, J / J_{s}\right)=v K_{l}\left(q, v, J / J_{s}\right)$, where $v$ is replaced everywhere by $\left(1-q^{2}\right)^{1 / 2}$ (see eq. [2.6]). Since different powers of $J / J_{s}$ have different coefficients in $K_{l}$ and $K_{c}$, the vector $\boldsymbol{\kappa}_{p}$ rotates in the $q-v$ plane during the growth to saturation. Different results are obtained in the unsaturated $\left(J / J_{s}=0\right)$ and saturated $\left(J / J_{s} \rightarrow \infty\right)$ limits, and a single type 1 solution for all intensities does not exist; if a proper physical solution is to exist, it must be able to evolve from the former to the latter.

\subsubsection{The $J=0$ Limit}

In this limit, the absorption coefficients of equation (4.4) are applicable for every value of $x_{B}$. The reason is that, in the absence of radiative interactions, the rate equations have an identical functional form for all Zeeman splittings. In this limit $K_{l}$ and $K_{c}$ are independent of $q$ and $v$ and the polarization solution can be obtained by inserting the appropriate terms from equation (4.4) directly into equation (2.6), yielding

$$
\begin{aligned}
& q=\frac{\left(R_{1}-1\right) \sin ^{2} \theta}{\sqrt{\left(R_{1}-1\right)^{2} \sin ^{4} \theta+4 R_{c}^{2} \cos ^{2} \theta}}, \\
& v=\frac{2 R_{c} \cos \theta}{\sqrt{\left(R_{1}-1\right)^{2} \sin ^{4} \theta+4 R_{c}^{2} \cos ^{2} \theta}} .
\end{aligned}
$$

This result provides the type 1 polarization at $J=0$ for any Zeeman splitting and can be used to follow the variation of polarization with magnetic field strength at any frequency and propagation direction. In particular, when $x_{B}>1$ these expressions should reproduce the solutions for the fully resolved Zeeman case, which are $J$-independent, at the three frequencies $v_{m}$. This is indeed the case. Comparison with equations (3.1) and (3.2) shows that the $\pi$-polarization is produced when $R_{1}=$ $R_{c}=0$, the $\sigma$-polarizations when both $R_{1}$ and $R_{c}$ greatly exceed unity with their ratio obeying $R_{1} / R_{c}= \pm 1$. Indeed, these are the respective behaviors of $R_{1}$ and $R_{c}$ when $x_{B}>1$. Consider first the $\pi$-component. In this case, both $R_{1}$ and $R_{c}$ vanish across the entire line $(|x| \lesssim 1)$ for large $x_{B}$, as can be seen from equation (4.2): at $x=0, R_{c}$ vanishes for any $x_{B}$ and $R_{1}=\exp \left(-x_{B}^{2}\right)$, vanishing rapidly when $x_{B}>1$; and at $|x|=1$, both $R_{1}$ and $R_{c}$ vanish faster than $\exp \left(-x_{B}\right)$ when $x_{B}>2$. Therefore, the $\pi$-polarization is properly reproduced by equation (4.5) and is established at the line wings at slightly larger Zeeman splitting than at their centers, in agreement with the conclusion of the previous section. And at the frequencies of the $\sigma$-components,

$$
\begin{aligned}
& x=\mp x_{B}: \\
& \quad R_{1}=\frac{1}{2} e^{x_{b}^{2}}\left(1+e^{-4 x_{b}^{2}}\right), \quad R_{c}= \pm \frac{1}{2} e^{x_{b}^{2}}\left(1-e^{-4 x_{b}^{2}}\right) .
\end{aligned}
$$

Therefore, both ratios increase rapidly and obey $R_{1} / R_{c}= \pm 1$ when $x_{B}>1$, properly reproducing the $\sigma$-polarizations of equation (3.2).

Proceed now to $x_{B}<1$. The polarization properties of the solution are quite different in this regime because both $R_{1}-1$ and $R_{c}$ are small quantities in this case. With the aid of equation (4.2), expansion to second order in $x_{B}$ yields

$$
\begin{aligned}
& q \simeq \frac{-x_{B}\left(1-2 x^{2}\right) \sin ^{2} \theta}{\sqrt{x_{B}^{2}\left(1-2 x^{2}\right)^{2} \sin ^{4} \theta+16 x^{2} \cos ^{2} \theta}}, \\
& v \simeq \frac{-4 x \cos \theta}{\sqrt{x_{B}^{2}\left(1-2 x^{2}\right)^{2} \sin ^{4} \theta+16 x^{2} \cos ^{2} \theta}} .
\end{aligned}
$$

When $4|x| \cos \theta>x_{B} \sin ^{2} \theta$, the polarization is virtually purely circular, $v \approx \pm 1$, with the opposite sense in the two halves of the line. ${ }^{3}$ The transition from one sense of circular polarization to the other occurs over the central frequency region $4|x| \cos \theta<x_{B} \sin ^{2} \theta$, where the polarization is purely linear, $q \approx-1$. This fully polarized configuration requires precise phase relations among the three Zeeman components. Since the three transitions are pumped independently, this solution is unstable against perturbations, a conclusion confirmed by formal stability analysis.

\footnotetext{
${ }^{3}$ By contrast, pure $\sigma$-components generate $\theta$-dependent elliptical polarization (eq. [3.2]).
} 


\subsubsection{The Saturated Limit}

The polarization relevant for observations involves the saturated limit, $J / J_{s} \gg 1$, and can be obtained by retaining only the terms quadratic in $J / J_{s}$ of the equation $q K_{c}=v K_{l}$. From the explicit form of the resulting fourth order equation, an overall term $q-q_{I}$ factors out where

$$
\text { I: } \quad \begin{aligned}
q_{I} & =-\frac{\sin ^{2} \theta}{1+\cos ^{2} \theta}, \\
v_{I} & = \pm \frac{2 \cos \theta}{1+\cos ^{2} \theta} .
\end{aligned}
$$

This can be recognized as the $\sigma$-polarization in the fully resolved Zeeman case but with the opposite sign for $q$ (see eq. [3.2]), providing finite circular polarization that is independent of both $x_{B}$ and $x$. From symmetry this is impossible, so this is obviously a spurious solution. Indeed, there is no path to this polarization from the $J=0$ limit.

After factoring out the term $q-q_{I}$, the remainder is a cubic equation for $q$. All three roots of this equation involve the square root of a negative term so there are no physical solutions; type 1 polarization is impossible now. In each case, the coefficient of the imaginary part is proportional to $R_{c}$, which vanishes at $x_{B}=0$. Therefore, at this unphysical limit the imaginary parts are removed, producing the three type 1 solutions

$$
\begin{array}{llll}
\text { II }: & q=\frac{1+3 \cos ^{2} \theta}{3\left(1+\cos ^{2} \theta\right)}, & v= \pm \frac{2\left(2+3 \cos ^{2} \theta\right)^{1 / 2}}{3\left(1+\cos ^{2} \theta\right)} \\
\text { III: } & q=+1, & v=0 \\
\text { IV: } & q=-1, & v=0 .
\end{array}
$$

Indeed, these solutions were identified in previous studies, which were always conducted at the $x_{B}=0$ limit, and were shown to be unstable against perturbations. Solution II was found by GKK (their eq. [61]), who showed it to be unstable for all $\boldsymbol{\theta}$. Solution III was found in Paper II (eq. [2.3]) and likewise was shown to be unstable for all $\theta$. Solution IV was first derived by GKK (eq. [60]), who found it to be unstable for $\sin ^{2} \theta>\frac{1}{3}$ but stable for $\sin ^{2} \theta<\frac{1}{3}$. However, Paper II showed this solution to be unstable also for $\sin ^{2} \theta<\frac{1}{3}$ in the unsaturated domain, prohibiting its growth. The present general analysis, which is not restricted to any particular value of $x_{B}$, shows that all three are simply the real parts of spurious solutions whose imaginary parts vanish only in the unphysical limit $x_{B}=0$. These polarizations are not realized at any finite value of $x_{B}$, however small.

In conclusion, there is no stationary type 1 solution that grows into the saturated regime when $x_{B}<1$. Just as type 0 polarization is impossible when the Zeeman pattern is fully resolved, type 1 polarization is impossible for overlapping Zeeman components.

\subsection{Type 0 Polarization}

The type 0 solution can be derived from equation (2.7) or, equivalently, equation (2.8). The latter is more convenient to solve. When the absorption coefficients obey $\kappa^{+}=\kappa^{-}=\kappa^{0}=$ $\kappa_{m}$, the general steady-state result of equation (A6) becomes simply

$$
\kappa_{m}\left[1+\left(1+f_{\Delta m}\right) J / J_{s}\right]=\kappa_{0}^{\Delta m}
$$

for each $\Delta m$ transition. Adding up these three equations pro- duces the common growth rate of the four Stokes parameters,

$$
\kappa_{m}=\kappa_{0}^{0} \frac{\left(2 R_{1}+1\right) / 3}{1+(4 / 3) J / J_{s}} \simeq \kappa_{0}^{0} \frac{1-(2 / 3) x_{B}^{2}\left(1-2 x^{2}\right)}{1+(4 / 3) J / J_{s}} .
$$

This is a $\theta$-independent growth rate similar to that of a scalar maser with the same pumping scheme; note that $\kappa_{0}^{0}\left(2 R_{1}+1\right) / 3=\frac{1}{3}\left(\kappa_{0}^{+}+\kappa_{0}^{-}+\kappa_{0}^{0}\right)$. With this result, the three structure factors become

$$
f_{\Delta m}=\frac{4 \kappa_{0}^{\Delta m} / \kappa_{0}^{0}}{2 R_{1}+1}\left(1+\frac{3}{4 J / J_{s}}\right)-1-\frac{1}{J / J_{s}} .
$$

These factors directly determine the Stokes parameters, $q$ is determined from $f_{0}, v$ from the difference $f_{+}-f_{-}$(see eq. [A5]). The result is

$$
\begin{aligned}
& q=1-\frac{2}{\left(2 R_{1}+1\right) \sin ^{2} \theta}\left[3-2 R_{1}+\frac{2\left(1-R_{1}\right)}{J / J_{s}}\right], \\
& v=\frac{8 R_{c}}{\left(2 R_{1}+1\right) \cos \theta}\left(1+\frac{3}{4 J / J_{s}}\right) .
\end{aligned}
$$

Since the three absorption coefficients $\kappa^{\Delta m}$ are different from each other when $J \rightarrow 0$, obviously the type 0 condition cannot be obeyed in that limit. A fully stationary polarization is impossible during early stages of maser growth, and this is the reason why both $q$ and $v$ of the formal solution contain $J$ dependent terms. However, as seen from equation (4.4), relative differences among absorption coefficients at $J=0$ are only of order $x_{B}$ at most and can be overcome when radiative interactions become important. Indeed, the $J$-dependent terms in equation (4.13) vanish at high intensities and can be neglected in the expression for $q$ when $J / J_{s}>2\left|1-R_{1}\right| \simeq 2 x_{B}^{2}\left|1-2 x^{2}\right|$, for $v$ when $J / J_{s}>\frac{3}{4}$. Once these conditions are obeyed, the $J$-independent parts of the solution properly describe stationary polarization. Expanding the ratios $R_{1}$ and $R_{c}$ to second order in $x_{B}$, the stationary polarization solution is

$$
\begin{aligned}
& q=1-\frac{2}{3 \sin ^{2} \theta}\left[1+\frac{8}{3} x_{B}^{2}\left(1-2 x^{2}\right)\right], \\
& v=-\frac{16 x x_{B}}{3 \cos \theta} .
\end{aligned}
$$

The inverse dependence of $v$ on $\cos \theta$ reflects the fact that the circular polarization enters into the problem only as $w=$ $\frac{1}{2} v \cos \theta$ (see eq. [A5]).

Physical polarizations must always obey $q^{2}+v^{2} \leq 1$. Applied to the stationary solution, this inequality translates into a quadratic equation for $\sin ^{2} \theta$, placing bounds on the allowed propagation directions

$$
\frac{1}{3}\left[1+\frac{8}{3} x_{B}^{2}\left(1+2 x^{2}\right)\right] \leq \sin ^{2} \theta \leq 1-32 x_{B}^{2} x^{2} .
$$

Figure 2 displays these bounds plotting the largest and smallest allowed angles as functions of $x_{B}$ for various frequency shifts. At each of the marked values of $x$, physical solutions are possible only inside the $\theta-x_{B}$ region bounded by the corresponding curves. At $x_{B}=0$ these bounds yield only the constraint $\sin ^{2} \theta \geq \frac{1}{3}$, i.e., $\theta>35^{\circ}$, familiar from the GKK study. However, at any physical value of $x_{B}$ the upper bound is also meaningful, and propagation perpendicular to the magnetic axis is forbidden (except for line center). As $x_{B}$ increases, the lower bound on $\sin ^{2} \theta$ increases and the upper bound decreases, causing the allowed region of propagation direc- 


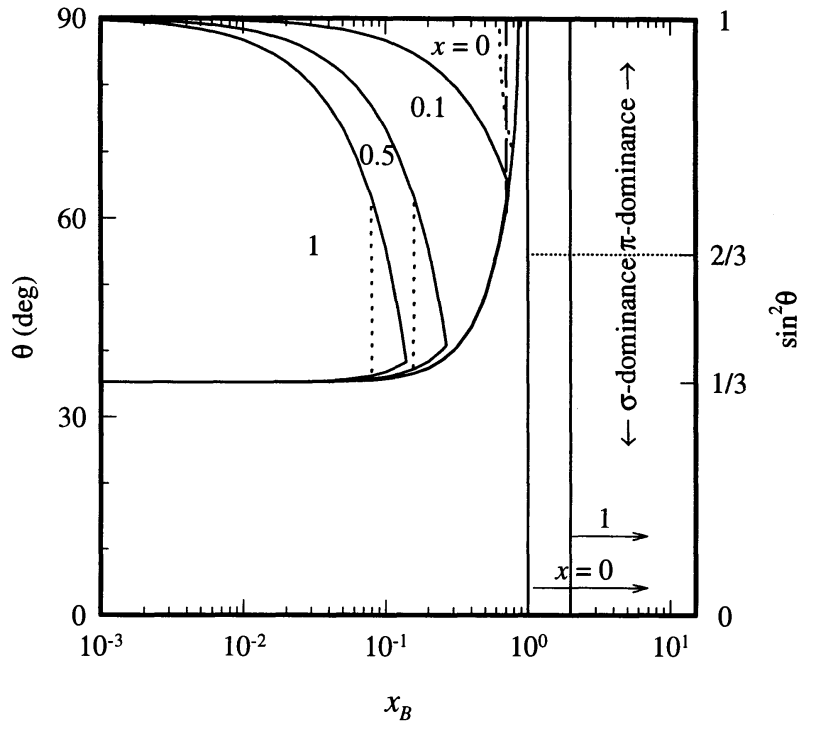

FIG. 2.-Phase space domains of physical solutions in a magnetic field with Zeeman splitting $x_{B}$ (eq. [1.1]). At each indicated frequency shift $x=(v$ $\left.-v_{0}\right) / \Delta v_{\mathrm{D}}$, polarized maser propagation is allowed only inside the $\theta-x_{B}$ region enclosed by the corresponding boundary. The domain for each $x$ contains all the domains for larger values of $x$. The primary bounds when $x_{B}<1$ are from eq. (4.15) and are denoted with full lines. At some frequencies they are supplemented by the bounds of eq. (4.3) (long-dashed line) and eq. (4.17) (shortdashed lines), assuming $\tau_{s}=15$. An absolute lower limit on $x_{B}$ for all frequencies, dependent on the pumping scheme and not plotted, is provided by eq. (4.18). When $x_{B}>1$, propagation is allowed for $x_{B}>1+x$ and all directions. The thin dotted line in this region marks the separation between directions where $\pi$ - and $\sigma$-components dominate.

tions to shrink. Finally, a physical solution is no longer possible when the two bounds coincide, and the radiation is unpolarized across the entire line for all propagation directions.

As discussed before, strictly stationary polarization is impossible during the early stages of maser growth. From the formal solution of the radiative transfer problem (eq. [2.11]), the amount by which any polarization varies during maser growth is controlled by the magnitude of the off-diagonal optical depth $\tau_{\pi}$ for that polarization. A polarization $\boldsymbol{\Pi}$ is approximately stationary if $\tau_{\pi}$ for that polarization is small. For the polarization solution of equation (4.14), $\tau_{\pi}$ is related to the diagonal optical depth $\tau_{m}$ through

$$
\tau_{\pi}=\frac{1}{3} \tau_{m} x_{B}^{2}\left[1+30 x^{2}+3\left(x^{2}-\frac{1}{2}\right) \sin ^{2} \theta\right] .
$$

This result is valid to order $x_{B}^{4}$ in the entire unsaturated domain, $J / J_{s}<1$, the region where optical depths grow linearly with path length. It shows that for radiation polarized according to the polarization solution, $\tau_{\pi}$ is much smaller than $\tau_{m}$, the optical depth that controls the exponential growth of the Stokes parameters. To a good degree of approximation, the polarization can be considered stationary if $\left|\tau_{\pi}\right|$ remains less than unity for the entire unsaturated growth phase. This phase is characterized by $\tau_{m}=\tau_{s}$, where $\tau_{s}$ is the optical depth that brings saturation, thus the polarization solution is valid so long as $\left|\tau_{\pi}\right|<1$ for $\tau_{m}=\tau_{s}$, or

$$
x_{B}^{2}\left|1+30 x^{2}+3\left(x^{2}-\frac{1}{2}\right) \sin ^{2} \theta\right| \lesssim \frac{3}{\tau_{s}} .
$$

This constraint ensures that the solution is approximately stationary also for the early growth phase. Figure 2 displays the bounds imposed by this constraint, too, for $\tau_{s}=15$, which is typical of astronomical masers' pumping schemes. As is evident from this figure, which plots also the bound of equation (4.3), the primary bound at all frequencies is provided by equation (4.15), with the other two bounds providing further minor restrictions at various frequencies. If we require propagation up to $\theta \simeq 80^{\circ}$ at all frequencies $|x| \leq 1$, then the Zeeman splitting allowed for type 0 polarization is limited to $x_{B} \lesssim 0.03$.

\subsubsection{Source Terms and Onset of the Solution}

The polarization solution cannot be established before the $J$-dependent terms start dominating the behavior of $K_{l}$. This occurs when the term linear in $J$ becomes equal to the $J$ independent term, i.e., $J / J_{s} \simeq x_{B}^{2}$ (see eq. [4.4]). However, the term that eventually controls the behavior of $K_{l}$ once the maser saturates is the one proportional to $\left(J / J_{s}\right)^{2}$. Indeed, the stationary polarization solution reflects the coefficient of this term and thus cannot be established before it too becomes comparable to the $J$-independent term, i.e., $\left(J / J_{s}\right)^{2} \simeq x_{B}^{2}$. Thus the solution requires $J / J_{s} \gtrsim x_{B}$.

Source terms were ignored thus far. Pumping of astronomical masers typically has $S_{0} / J_{s} \sim 10^{-5}$ to $10^{-8}$, thus the maser intensity greatly exceeds the source function $S_{0}$ at early stages of growth, long before saturation. However, the polarization solution reflects the behavior of the off-diagonal terms in the transfer of self-amplified radiation while the source terms involve the diagonal absorption coefficient $\kappa_{m}$. Thus the onset of the linear polarization solution requires $\kappa_{l} I>\kappa_{m} S_{0}$, or, since $\kappa_{m} \simeq 1$ in the entire unsaturated domain, $\kappa_{l} I / J_{s}>S_{0} / J_{s}$. In linear masers this condition is equivalent to $\kappa_{l} J / J_{s}>S_{0} / J_{s}$, in three dimensions there is an additional factor of $\Omega / 4 \pi$, where $\Omega$ is the beam solid angle. This condition must be obeyed when the $J$-dependent terms dominate the behavior of $\kappa_{l}$, at which time $\kappa_{l}$ is of order $J / J_{s}$. Thus the dominance of self-amplified radiation requires $\left(J / J_{s}\right)^{2}>S_{0} / J_{s}$, a condition first noted in Paper II. For this condition to be obeyed when the quadratic term surpasses the $J$-independent term $\left(J / J_{s} \simeq x_{B}\right)$, the Zeeman splitting must obey $x_{B}^{2}>S_{0} / J_{s}$; weaker fields will produce only unpolarized radiation. Combining these results yields

$$
J / J_{s} \gtrsim x_{B}>\sqrt{S_{0} / J_{s}} .
$$

For a given pumping scheme, these are the general bounds that must be obeyed by the Zeeman splitting and intensity to enable the polarization solution.

\section{ANISOTROPIC PUMPING}

An alternative to magnetic fields in generating maser polarization is anisotropic interaction rates, where a quantization axis is introduced by the pumping process itself. Among the concrete examples proposed are collisions with an electron stream (Johnston 1967). Because the pumping is then $m$ dependent it directly introduces distinctions among magnetic sublevels even though they remain strictly degenerate. As long as symmetry is maintained around the axis, $\kappa^{+}=\kappa^{-}=\kappa^{1}$ and $\kappa_{c}=R_{c}=v=0$. Circular polarization is impossible under these circumstances.

Consider the $1 \rightarrow 0$ maser model. The absorption coefficients are derived in the same manner as for overlapping Zeeman components but the ratio $R_{1}$ is now frequency-independent, 
given by

$$
R_{1}=\frac{P_{1,1}-P_{0}}{P_{1,0}-P_{0}} .
$$

Here $P_{1,1}$ denotes the common pump rate of the $m= \pm 1$ sublevels. From the bounds of equation (A12), $R_{1}$ must obey $1 / 2<R_{1}<3 / 2$ whenever all three transitions are radiatively coupled, to ensure that they remain inverted in the saturated domain. In addition, $R_{1}=1$ is unphysical because an axis cannot be defined in that case. However, similar to $x_{B}=0$ for magnetic fields, this value can be approached as a limit since arbitrarily small $\left|1-R_{1}\right|$ correspond to physical situations. The absorption coefficients again can be written as $\kappa^{\Delta m}=$ $\kappa_{0}^{0} K^{\Delta m} / D$, and the corresponding expressions for $D$ and $K^{\Delta m}$ are simpler than in the case of Zeeman splitting. The relevant analogs of equation (4.4) are

$$
\begin{aligned}
D & =1+\left(2-f_{1}\right) J / J_{s}+4 f_{0} f_{1}\left(J / J_{s}\right)^{2}, \\
K_{l} & =\frac{1}{2} \sin ^{2} \theta\left\{R_{1}-1+\left(J / J_{s}\right)\left[2 R_{1}\left(1-f_{1}\right)-1-f_{1}\right]\right\} .
\end{aligned}
$$

\subsection{Type 1 Polarization}

Because $\kappa_{c}=0, \kappa_{p}=\left|\kappa_{l}\right|$ and from equation (2.6), the type 1 polarization is

$$
q=\frac{\kappa_{l}}{\left|\kappa_{l}\right|}=\frac{K_{l}}{\left|K_{l}\right|}= \pm 1 .
$$

Self-consistency requires $K_{l}$ to have the same sign as $q$. Since the $J$-independent term of $K_{l}$ is proportional to $R_{1}-1$ (eq. [5.2]), unsaturated growth is possible for $q=+1$ polarization only when $R_{1}>1$, for $q=-1$ when $R_{1}<1$; the discontinuous transition between the two solutions occurs at the singular point $R_{1}=1$. Furthermore, $K_{l}$ must maintain its sign after the maser saturates.

Consider first the $q=+1$ solution. In this case $f_{1}=\frac{1}{2}$ and $K_{l}$ remains positive after saturation only if $R_{1}>3 / 2$. Although the right-hand inequality of equation (A12) is violated in that case, the solution is still valid. That particular inequality reflects the requirement that the $\Delta m=0$ transition remain inverted in the saturated domain. But this requirement does not apply here because $q=+1 \operatorname{implies} f_{0}=0$, and the $\Delta m=0$ transition does not couple radiatively. This polarization is generated purely by the $|\Delta m|=1$ transitions; indeed its growth rate is

$$
\lambda=\kappa_{m}+\kappa_{l}=\frac{\kappa_{0}^{1}}{1+(3 / 2) J / J_{s}},
$$

where $\kappa_{0}^{1}\left(=\kappa_{0}^{0} R_{1}\right)$ is the unsaturated limit of $\kappa^{1}$.

The $q=-1$ polarization grows when $\frac{1}{2}<R_{1}<1$ and produces $f_{0}=\sin ^{2} \theta, f_{1}=\frac{1}{2} \cos ^{2} \theta$. The requirement that $K_{l}$ remain negative after saturation does not further constrain $R_{1}$; instead it restricts the propagation directions to

$$
\sin ^{2} \theta<\frac{3-2 R_{1}}{2 R_{1}+1} .
$$

This range in displayed in Figure 3. At the singular upper limit $R_{1}=1$, propagation is allowed only for $\sin ^{2} \theta<\frac{1}{3}$. As $R_{1}$ decreases, the range of allowed propagation directions increases until it encompasses all angles at the lower limit

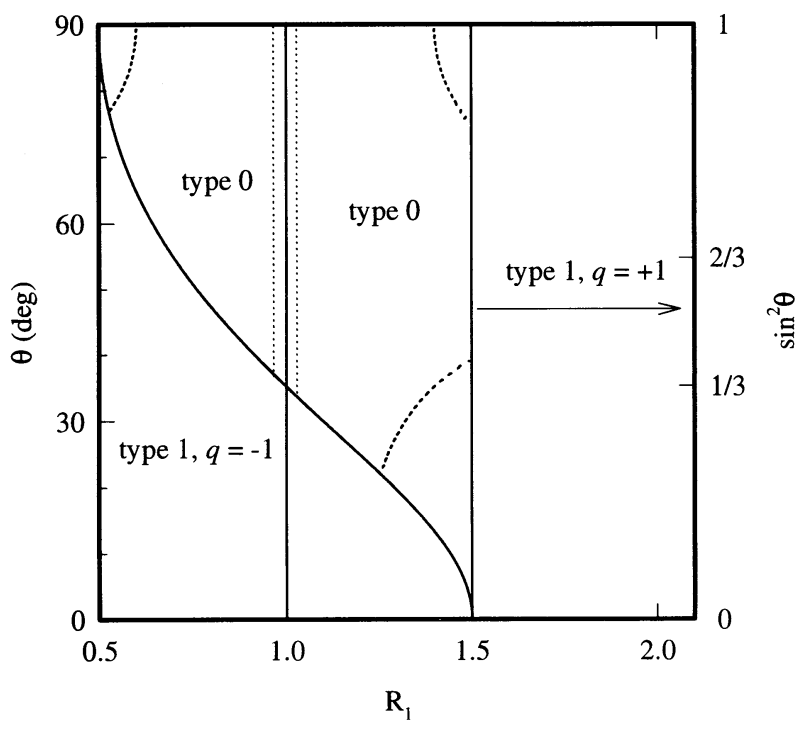

FIG. 3.-Phase space domains of physical solutions for $m$-dependent pumping whose degree of anisotropy is characterized by $R_{1}$ (eq. [5.1]). The solutions applicable in the different regions are listed in Table 2 . The shortdashed lines that further restrict the corners of the domains of type 0 polarization are from eq. (5.9), assuming $\tau_{s}=15$. The dotted lines on the two sides of $R_{1}=1$ are a schematic representation of the bound of eq. (5.10). The actual location of these lines depends on the pumping scheme.

$R_{1}=\frac{1}{2}$. This polarization involves all three transitions and its growth rate is

$$
\begin{aligned}
\lambda & =\kappa_{m}-\kappa_{l} \\
& =\kappa_{0}^{0} \frac{2\left(\sin ^{2} \theta+R_{1} \cos ^{2} \theta\right)+(1 / 4) \sin ^{2} 2 \theta J / J_{s}}{2+\left(3+\sin ^{2} \theta\right) J / J_{s}+\sin ^{2} 2 \theta\left(J / J_{s}\right)^{2}} .
\end{aligned}
$$

As with the polarization in a magnetic field, once the maser saturates for propagation at a given direction, the rate of further growth in the saturated domain is angle-independent.

\subsection{Type 0 Polarization}

Since this polarization involves all three transitions, $R_{1}$ must adhere to the bound of equation (A12), $\left|1-R_{1}\right|<\frac{1}{2}$. Except for the absence of circular polarization, this solution is identical to that for overlapping Zeeman components (see eqs. [4.11] and [4.13]). The $J$-dependence of $q$ can be neglected when $J / J_{s}>2\left|1-R_{1}\right|$, the resulting stationary polarization is

$$
q=1-\frac{2\left(3-2 R_{1}\right)}{\left(2 R_{1}+1\right) \sin ^{2} \theta} .
$$

At $R_{1}=3 / 2$, the upper limit allowed for this solution, it produces $q=+1$, same as the type 1 solution that takes over at that value of $R_{1}$. The requirement $|q| \leq 1$ constrains the propagation directions to

$$
\sin ^{2} \theta \geq \frac{3-2 R_{1}}{2 R_{1}+1}
$$

the complementary angular range of the type 1 solution $q=-1$ (see eq. [5.5] and Fig. 3). At their common phase-space boundary, these two polarizations are the same. As with the analogous case of overlapping Zeeman components, this polarization is not stationary at low intensities. The requirement that $\left|\tau_{\pi}\right|$ be smaller than 1 when $\tau_{m}=\tau_{s}$ produces the 
TABLE 1

Polarization Solutions-Magnetic Field

\begin{tabular}{|c|c|c|c|c|}
\hline Validity Domain & Type & $q$ & $v$ & $\lambda$ \\
\hline \multicolumn{5}{|l|}{$\begin{array}{l}\sqrt{S_{0} / J_{s}}<x_{B}<1 / \sqrt{2} \\
J / J_{s}>x_{B} \\
\sin ^{2} \theta>\frac{1}{3}\left[1+\frac{8}{3} x_{B}^{2}\left(1+2 x^{2}\right)\right]\end{array}$} \\
\hline $\cos \theta>4 \sqrt{2} x x_{B}^{\mathrm{a}} \ldots \ldots \ldots \ldots$ & 0 & $1-\frac{2\left[1+(8 / 3) x_{B}^{2}\left(1-2 x^{2}\right)\right]}{3 \sin ^{2} \theta}$ & $-\frac{16 x x_{B}}{3 \cos \theta}$ & ${\frac{\kappa_{0}^{0}}{1+(4 / 3) J / J_{s}}}^{b}$ \\
\hline$x_{B}>1+x ; \pi \ldots \ldots \ldots \ldots \ldots \ldots$ & 1 & -1 & 0 & $\kappa \sin ^{2} \theta^{c}$ \\
\hline$x_{B}>1+x ; \sigma^{ \pm} \ldots$. & 1 & $\frac{\sin ^{2} \theta}{1+\cos ^{2} \theta}$ & $\pm \frac{2 \cos \theta}{1+\cos ^{2} \theta}$ & $\frac{1}{2} \kappa\left(1+\cos ^{2} \theta\right)^{\mathrm{c}}$ \\
\hline
\end{tabular}

NoTE--Polarization solutions for masers in magnetic fields characterized by Zeeman splitting $x_{B}$ (eq. [1.1]) The different types of polarization solutions are discussed in $\S 2.1 .1$.

a These are the primary constraints on the $\theta-x_{B}$ phase space for this solution (eqs. [4.3], [4.15], and [4.18]). Additional constraints are provided by eq. [4.17])

b These results hold for all spins, with $\kappa_{0}^{0}$ the absorption coefficient in the absence of both Zeeman splitting and maser radiative interaction.

c These results hold for all spins, with $\kappa$ the absorption coefficient of the transition. Detailed expressions displaying the explicit dependence of $\kappa$ on $J / J_{s}$ are listed for $1 \rightarrow 0$ transitions in equation (3.4).

constraint

$$
\left|\left(1-R_{1}\right)\left(\sin ^{2} \theta-\frac{2}{3}\right)\right|<\frac{2}{\tau_{s}} .
$$

Figure 3 displays the bounds produced by this constraint for $\tau_{s}=15$ when they further restrict the phase space for physical solutions. Finally, the requirement that the source function be negligible when the $J$-dependent term dominates the behavior of $K_{l}$ is

$$
J / J_{s} \gtrsim\left|1-R_{1}\right|>\sqrt{S_{0} / J_{s}}
$$

the analogue of equation (4.18).

\section{SUMMARY AND DISCUSSION}

The results presented here provide the general solutions for maser polarization in all cases that have been addressed in the literature thus far. Table 1 summarizes the properties of the solutions for any magnetic field strength; Table 2 does the same for $m$-dependent pumping with any degree of anisotropy. These tabulations are further aided by Figures 2 and 3 which display the phase space regions where each solution is applic- able. Appendix B provides an analysis of the phase relations obeyed by the electric fields of the polarization solutions.

\subsection{Comparison with Previous Studies}

Previous detailed studies concentrated mostly on maser polarization in a magnetic field and were conducted in one of two limits, either $x_{B} \rightarrow \infty$ or $x_{B}=0$. The results of $\S 3$ fully corroborate those of previous studies in the first limit (GKK, Paper I). For any spin, this polarization is established at a given frequency once the Zeeman pattern is resolved at that frequency, the approach to the limit solution is at least exponential in $x_{B}$. The polarization is established at the line centers of the Zeeman components when $x_{B} \gtrsim 1$ and takes hold over the entire spectral region of interest when $x_{B} \gtrsim 2$. A significant new result involves the absorption coefficients for $1 \rightarrow 0$ transitions (eq. [3.4]). These show a considerable degree of anisotropy, introducing substantial differences between the behavior of the $\pi$ - and $\sigma$-components at most angles.

Overlapping Zeeman components were previously studied only at the unphysical limit $x_{B}=0$. The $x_{B} \rightarrow 0$ limit of the solution derived here $(\S 4.2)$ verifies the linear polarization found in the previous studies (GKK; Papers I, II). However,

TABLE 2

Polarization Solutions-Anisotropic Pumping

\begin{tabular}{lccc}
\hline \multicolumn{1}{c}{ Validity Domain } & Type & $q$ & $\lambda$ \\
\hline$\frac{1}{2}<R_{1}<1 ;$ & & -1 & $\kappa_{0}^{0} \frac{2\left(\sin ^{2} \theta+R_{1} \cos ^{2} \theta\right)+(1 / 4) \sin ^{2} 2 \theta J / J_{s}}{2+\left(3+\sin ^{2} \theta\right) J / J_{s}+\sin ^{2} 2 \theta\left(J / J_{s}\right)^{2}}$ \\
$\sin ^{2} \theta<\frac{3-2 R_{1}}{2 R_{1}+1} \ldots \ldots$. & 1 & & \\
$\sqrt{S_{0} / J_{s}}<\left|1-R_{1}\right|<\frac{1}{2} ;$ & & & $\frac{\kappa_{0}^{0}\left(2 R_{1}+1\right) / 3}{1+(4 / 3) J / J_{s}}$ \\
$J / J_{s}>2\left|1-R_{1}\right| ;$ & & $1-\frac{2\left(3-2 R_{1}\right)}{\left(2 R_{1}+1\right) \sin ^{2} \theta}$ & $\frac{\kappa_{0}^{1}}{1+(3 / 2) J / J_{s}}$ \\
$\sin ^{2} \theta>\frac{3-2 R_{1}}{2 R_{2}+1} \ldots \ldots .$. & 0 & +1 & 1
\end{tabular}

NOTE.-Polarization solutions for masers in the case of pumping with a degree of anisotropy characterized by $R_{1}$ (eq. [5.1]). $\kappa_{0}^{0}$ is the unsaturated absorption coefficient of the $\Delta m=0$ transition, $\kappa_{0}^{1}$ that of $|\Delta m|=1$. 
while terms of higher order in $x_{B}$ have an insignificant effect on the magnitude of $q$, they produce some major new results.

\subsubsection{Circular Polarization}

The linear polarization is accompanied by circular polarization, proportional to $x_{B}$ (eq. [4.14]). For saturated masers, $I$ follows the Gaussian frequency profile of the unsaturated absorption coefficients and the Stokes parameter $V(=v I)$ is

$$
V(x)=\frac{4 I(0) x_{B}}{3 \cos \theta} F\left(x ; x_{B}\right) .
$$

Here $I(0)$ is the intensity at line center and

$$
F\left(x ; x_{B}\right)=\frac{1}{x_{B}}\left[e^{-\left(x+x_{B}\right)^{2}}-e^{-\left(x-x_{B}\right)^{2}}\right] \simeq-4 x e^{-x^{2}}
$$

is the frequency profile of the circular polarization. The last approximation is for $x_{B}<1$, corrections are only of order $x_{B}^{2}$. Thus the profile is independent of $x_{B}$ to a high degree of accuracy in the entire region of interest, and is plotted in Figure 4. The two peaks of the function occur at $x= \pm 1 / 2^{1 / 2}$ and the magnitude at the peaks is $(8 / e)^{1 / 2}$. As the magnetic field strength varies, the profile of $V$ is simply scaled by $x_{B}$. The frequency separation of the two peaks, $2^{1 / 2} \Delta v_{\mathrm{D}}$, remains fixed and can be used to determine the Doppler width, but not the field strength. Once the Doppler width is known, the Zeeman splitting can be determined from $v_{\text {peak }}$, the ratio $V / I$ at the peak of the Stokes parameter $V$, through

$$
x_{B}=\frac{3 \sqrt{2}}{16} v_{\text {peak }} \cos \theta \text {. }
$$

As usual, because of the uncertainty about the direction of the field, its magnitude cannot be uniquely established. Since the spectra of the Stokes parameters obey $V \propto d I / d x$, the magnetic field can also be determined using the method described by Troland \& Heiles (1982) for data analysis when $x_{B}<1$.

The circular polarization is responsible for an upper limit on the directions allowed for polarized maser radiation, and propagation perpendicular to the magnetic field is forbidden. The largest direction possible at Zeeman splitting $x_{B}$ and frequency

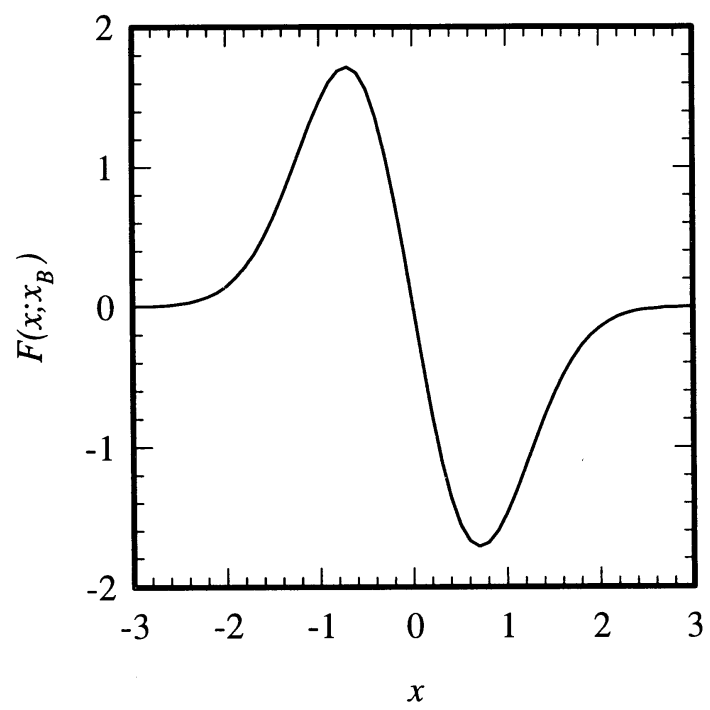

Fig. 4.-Profile of the circular polarization when $x_{B} \ll 1$ (eq. [6.2]) $x$ is $\theta=\cos ^{-1}\left[4(2)^{1 / 2} x x_{B}\right]$, the upper bound displayed in Figure 2 for some frequencies $x$. Propagation along this boundary of allowed phase-space produces $v=0.94$. Except for the immediate vicinity of this extreme edge, $v$ is of order $x_{B}$ for most propagation directions.

\subsubsection{Onset of Linear Polarization}

In addition to introducing circular polarization, the higher order terms hold the key to some fundamental properties of maser polarization when $x_{B}<1$ that are not directly accessible to observations. At $x_{B}=0$, the $J$-dependence of the type 0 solution for $q$ disappears (see eq. [4.13]; note that $R_{1}=1$ in this limit), a singularity that caused considerable difficulty in previous studies because of the conceptual problem associated with polarization growth: Since the seed for maser radiation is spontaneous decays, when $x_{B}=0$ the radiation starts unpolarized with intensity $S_{0}$. And since the polarization solution does not introduce any additional intensity scale in this limit, it was not clear at which intensity this solution would become applicable. Attempts to overcome this difficulty involved introduction of extraneous intensity scales through various arguments. While GKK claimed that the polarization sets in only after the maser saturates $\left(J / J_{s}>1\right)$, Paper II argued that $J / J_{s} \gtrsim 1 / \tau_{s}$ was the more appropriate condition ( $\tau_{s}$ is the saturation optical depth).

The general analysis presented here finally resolves this issue - the linear polarization solution requires $J / J_{S} \gtrsim x_{B}>$ $\left(S_{0} / J_{s}\right)^{1 / 2}$ (eq. [4.18]). The reason is quite simple. This polarization is established when the average populations of the $m= \pm 1$ and $m=0$ magnetic substates of the maser upper level become equal to each other. In the presence of Zeeman splitting and the absence of interaction with maser radiation, these populations are different. Interactions with properly polarized maser radiation equalize the sublevel populations once these interactions dominate the pumping process. This occurs when $B J / \Gamma\left(=J / J_{s}\right)$, the radiative rate relative to the loss rate of the pumping scheme, exceeds $x_{B}$. By comparison, saturation is the process in which radiative interactions reduce the inversion itself, the difference between the average populations of the entire upper and lower levels, and requires $J / J_{s}>1$. The polarization solution requires amplification with an optical depth $\frac{1}{2} \tau_{s}$, halfway through the unsaturated domain.

The intensity scale of saturated maser radiation is determined by $J_{s}$; the larger is $J_{s}$ the more powerful the maser. For a given source function, equation (4.18) shows that the polarization solution requires $x_{B}^{2} J_{s}>S_{0}$-stronger masers can enable the polarization solution at smaller Zeeman splitting. Conversely, when the maser pumping becomes weaker at a fixed Zeeman splitting, this condition cannot be met and the polarization disappears; as the maser begins to resemble a thermal source, it becomes unpolarized.

\subsubsection{Spurious Solutions}

While the $x_{B} \rightarrow 0$ limit of the solution derived here reproduces the linear polarization of previous studies, there is one notable exception. None of the spurious solutions which required stability analysis for their removal in those studies is generated here. Indeed, as is evident from both Tables 1 and 2 and Figures 2 and 3, in any given region of phase space there is never more than one physical solution, if at all. In particular, the solution $q=-1$ whose stability properties for $\sin ^{2} \theta<\frac{1}{3}$ caused some problems is not the $x_{B} \rightarrow 0$ limit of any physical solution. Rather, it is obtained only as the $x_{B} \rightarrow 0$ limit of a 
spurious solution whose imaginary part vanishes in this unphysical limit (see $\S 4.1$, especially eq. [4.9]). There are no physical solutions for polarized maser radiation at $\sin ^{2} \theta<\frac{1}{3}$ when $x_{B}<1$, in agreement with the conclusion of Paper II.

It is worth noting that the results of a formalism that assumes $x_{B}=0$ at the outset cannot be distinguished from those of the equally unphysical limit $R_{1}=1$ of anisotropic pumping. Indeed, the latter case fully reproduces the GKK results. At $\sin ^{2} \theta<\frac{1}{3}$ it has $q=-1$ and at $\sin ^{2} \theta>\frac{1}{3}$ it has the same polarization as the $x_{B}=0$ formal limit of the magnetic case. Obviously, though, anisotropic pumping has no direct relevance to the behavior in a magnetic field, as is evident when the two models are compared at physical, i.e., finite values of $x_{B}$ and $\left|1-R_{1}\right|$, however small. This further underscores the inherent pitfalls in a formulation that assumes a limit from the outset instead of properly approaching that limit within a general framework.

\subsection{Polarization Variation with Field Strength}

With the dependence on $x_{B}$ explicitly included, the evolution of the polarization with magnetic field strength can be described. The bounds placed on the Zeeman splittings and angles that allow different physical solutions are displayed in Figure 2 and Table 1 . At $x_{B} \lesssim 10^{-3}$, the allowed physical solution corresponds to the type 0 polarization listed in equation (4.14), applicable for essentially all frequencies and $\sin ^{2} \theta>\frac{1}{3}$. As the field strength increases, the allowed angular region shrinks at a frequency-dependent rate that increases with distance from line center, thus the spectral region of allowed propagation shrinks inward and the line wings disappear first. This can lead to narrow maser features with linewidths less than the Doppler width in sources with $x_{B} \gtrsim 0.1$. Such features will display substantial circular polarization. Finally, when $x_{B} \gtrsim 0.7$ propagation is no longer possible for any direction even at line center, and the entire line is unpolarized.

All along, the type 1 polarization listed in equation (4.5) provides another allowed solution, albeit one that is unstable and that does not grow into the saturated regime so long as $x_{B}<1$. As the field strength increases further, these deficiencies are removed and this solution becomes the physical polarization listed in equations (3.1) and (3.2), first at the centers of the three Zeeman components when $x_{B}>1$, spreading to the line wings when $x_{B}>2$. This $x_{B}$-independent solution describes the physical polarization at all higher values of $x_{B}$.

\subsection{Higher Spins}

Whenever explicit expressions for the absorption coefficients were required, those of $J=1 \rightarrow 0$ transitions were utilized. Absorption coefficients for all other spins, which follow directly from general level population equations listed in Paper I, can be avoided in most applications because various properties of the solutions are independent of the transition spins. In particular, type 0 polarization is characterized by absorption coefficients $\kappa^{\Delta m}$ that are equal to each other (eq. [2.8]), and because of general properties of the vector-coupling coefficients, such solutions are spin-independent (Paper I). Thus the type 0 solutions, in particular the polarization in a magnetic field with $x_{B}<1$, are valid for all spins (see Papers I, II). Further, the polarizations of fully resolved Zeeman patterns (eqs. [3.1] and [3.2]) were derived without even specifying the absorption coefficients, thus they too are spin-independent. Only the detailed expressions for the absorption coefficients of the $\pi$ - and $\sigma$-components listed in equation (3.4) are based on explicit results for $1 \rightarrow 0$ transitions. These expressions are the starting point for analysis of the intensity angular distribution for the different Zeeman components, so this discussion strictly holds only when the $1 \rightarrow 0$ results are applicable. Included in this category are all transitions between equal spin states and $m$-independent pumping, because they share the same simple Zeeman pattern, so these results apply without modification to the $\mathrm{OH}$ main lines but not to its satellite lines. Still, the general trend of anisotropy is expected to hold in the latter case, too (note in particular the angular dependence of the general expressions for the growth rates in eqs. [3.1] and [3.2]).

It is important to emphasize the fundamental assumption that the only degeneracy of the transition levels involves their magnetic substates. When any of the maser levels includes additional degeneracy, so that magnetic substates of different levels overlap, the tight constraints responsible for the general solutions no longer apply and the polarization can be expected to disappear. This can be easily understood by considering the imaginary limit in which the hyperfine splitting of the $\mathrm{OH}$ molecule vanishes and its four ground-state maser lines blend into one. The polarization properties of masers with hyperfine degeneracy, notably $\mathrm{H}_{2} \mathrm{O}$ and methanol, cannot be studied without specifying the full details of their pumping schemes. The general solutions derived here apply to such masers only when they involve excitation of a single hyperfine transition.

\subsection{Comparison with Observations}

Since most results of the previous studies are reproduced here, the basic overall successes of maser polarization theory remain intact. $\mathrm{OH}$ is the only astronomical maser whose Zeeman pattern can be fully resolved with typical interstellar fields. It fulfills the condition $x_{B}>1$ for magnetic fields of milligauss, frequently measured in $\mathrm{H}$ II/OH regions. Indeed, these masers are properly described by the theory for resolved Zeeman patterns. The most detailed polarization measurements were done for $\mathrm{W} 3(\mathrm{OH})$ by Garcia-Barreto et al. (1988) and Bloemhof, Reid, \& Moran (1992), and are adequately explained if Faraday rotation is responsible for removal of some linear polarization. A significant new result is the great disparity, which presists into the saturated domain, between Zeeman components at different angular regions, resulting in a preponderance of $\sigma$-components $(\S 3)$. Garcia-Barreto et al. find that there are no features in $\mathrm{W} 3(\mathrm{OH})$ that might be identified as $\pi$-components, even accounting for possible Faraday rotation. The results of $\S 3$ adequately explain this observation if the magnetic field is aligned within less than $55^{\circ}$ from the line of sight.

For overlapping Zeeman components, the conclusions of Papers I and II regarding linear polarization are reproduced and the observational implications of these studies carry over. Maser polarization is established during the unsaturated exponential growth phase and is independent of spin. All purespin maser transitions of nonparamagnetic molecules should display the same polarization properties. This appears to be the case for $\mathrm{SiO}$ masers, which generally display high degrees of linear polarization. McIntosh \& Predmore $(1991,1993)$ have extended $\mathrm{SiO}$ linear polarization measurements up to $J=3 \rightarrow 2$ and their observations indicate that polarization properties indeed are $J$-independent-different lines display detailed similarities between fractional polarization, polarization position angle, and rotation of position angle with velocity. Faraday depolarization affects the low rotation states more because of their longer wavelengths and the polarization can 
be expected to decrease toward lower angular momenta, as observed. Although detailed calculations of Faraday rotation have yet to be performed, McIntosh \& Predmore (1993) find this to be the most plausible explanation of their data.

Unlike $\mathrm{SiO}$, which is a simple rotor, $\mathrm{H}_{2} \mathrm{O}$ maser transitions involve overlapping hyperfine components and the polarization can be expected to disappear in general. Indeed, $\mathrm{H}_{2} \mathrm{O}$ masers are generally only weakly polarized (e.g., Barvainis \& Deguchi 1989). Exceptions do exist, though, and $\mathrm{H}_{2} \mathrm{O}$ masers sometime display high polarization, notably during outbursts such as the one in Orion (Garay, Moran \& Haschick 1989; Abraham \& Vilas Boas 1994). Such cases may involve the excitation of a single hyperfine component. Similar to $\mathrm{H}_{2} \mathrm{O}$, methanol lines are in general split into many hyperfine components due to a number of mechanisms and can be expected to have polarization properties similar to $\mathrm{H}_{2} \mathrm{O}$. Indeed, this is what Koo et al. (1988) found in a methanol $12.2 \mathrm{GHz}$ survey. This transition has a spin-rotation splitting of only $\sim 2-3 \mathrm{kHz}$ (Gaines, Casleton, \& Kukolich 1974), a degeneracy that could be responsible for the similarity to $\mathrm{H}_{2} \mathrm{O}$ polarization properties.

A major new result is the circular polarization for $x_{B}<1$. The Stokes $V$ spectrum has the traditional Zeeman antisymmetric S-curve profile, plotted in Figure 4. Such spectra are not always observed. However, this profile follows directly from the general symmetry in left-right rotations about the magnetic axis, independent of the specific theory developed. While this symmetry cannot be broken with the quiescent medium and uniform magnetic field assumed here, the model can be supplemented with symmetry breaking in the form of magnetic and/or velocity gradients, as done in various filter mechanisms (e.g., Deguchi \& Watson 1986, and references therein). Such modifications can easily account for the removal of one component.

Circular polarization, at the level of a few percent, was discovered in $\mathrm{SiO}$ observations of late-type stars by Barvainis, McIntosh, \& Predmore (1987). Following their discussion we take $\theta \approx 45^{\circ}$ and denote the percent maximal circular polarization by $m_{c}\left(=100 v_{\text {peak }}\right)$, then equation (6.3) shows that $x_{B}=$ $2 \times 10^{-3} m_{c}$. Observed values of $m_{c}$ range from 1.5 to 9 , therefore $x_{B}$ varies from $3 \times 10^{-3}$ to $\sim 10^{-2}$. The magnetic field can then be determined from equation (1.1), which leads to $B=$ $2 m_{c} \Delta v_{5} \mathrm{G}$ for the $43 \mathrm{GHz}$ line. Invoking a filter mechanism proposed by Deguchi \& Watson (1986), Barvainis et al. devised an estimate for the magnetic field that has an identical expression, only the numerical coefficient is a factor of 8 larger. $^{4}$ Therefore, once their estimates for individual sources are scaled downward by an overall factor of 8 they hold as is, resulting in fields of $2-10 \mathrm{G}$.

When the transition frequency varies, the Doppler width varies proportionately while the Zeeman splitting remains fixed. Therefore $x_{B}$ is inversely proportional to frequency (see eq. [1.1]) and the circular polarization decreases with the transition frequency when all other properties remain fixed. The circular polarization of $\mathrm{SiO}$ masers can be expected to decrease when the rotation quantum number increases. Indeed, observations of VY CMa by McIntosh, Predmore, \& Patel (1994) show that the circular polarization of $J=2 \rightarrow 1$ is smaller than that of $J=1 \rightarrow 0$, the opposite of the trend displayed by linear polarization.

${ }^{4}$ Note that the Barvainis et al. expression is written in terms of full width at half-maximum.
Circular polarization is observed also in $\mathrm{OH}$ maser emission from late-type stars. In the case of supergiants it can be substantial-50\% and higher is common (e.g., Cohen et al. 1987, and references therein). In the absence of theory for $x_{B}<$ 1 , this was taken as a signature of $x_{B} \gtrsim 1$ and magnetic fields of at least a few milligauss. However, this polarization often appears as sharp reversals between adjacent narrow spectral components of the parameter $V$, similar to the profile displayed in Figure 4, thus the two circular components are not separated and the data must be analyzed according to the theory for $x_{B}<1$. The separation between the peaks cannot be used as an indication of the magnetic field strength, neither is a determination of Doppler width from a fit to individual components an adequate procedure. Instead, the Doppler width should be determined from the separation of the two peaks, the field strength from equation (6.3).

Since the $\mathrm{OH}$ circular polarization is typically an order of magnitude higher than for $\mathrm{SiO}$, the parameter $x_{B}$ is generally an order of magnitude larger. Assuming for simplicity $\theta \approx 45^{\circ}$, equation (6.3) shows that $50 \%$ circular polarization corresponds to $x_{B} \simeq 0.1$, consistent with overlapping Zeeman components. From Figure 2, at such a large $x_{B}$ propagation at $45^{\circ}$ is forbidden at $x=1$ and is allowed only closer to line center. This illustrates the importance of constraints at such high values of $x_{B}$ and may help explain the narrowness of observed components in the profile of $V$. For the $1665 \mathrm{MHz}$ line, $x_{B}=$ 0.1 requires a field of only 0.4 milligauss for a Doppler width of $1 \mathrm{~km} \mathrm{~s}^{-1}$. For any polarization, the field can be obtained from $B=0.7 v_{\text {peak }} \Delta v_{5} \mathrm{mG}$, and it is only $\sim 0.1 \mathrm{mG}$ for typical parameters. Thus the magnetic fields in supergiant maser regions are one to two orders of magnitudes lower than what was previously inferred in the literature (e.g., Reid et al. 1979; Claussen \& Fix 1982; Cohen et al. 1987). In contrast, maser emission from $\mathrm{OH} / \mathrm{IR}$ stars displays somewhat lower circular polarization ( $\lesssim 15 \%$ ), which was properly analyzed by Zell \& Fix (1991) with the Troland \& Heiles (1982) method. This analysis yielded estimates of field strengths less than $0.1 \mathrm{G}$, and those remain valid.

Circular polarization with $v_{\text {peak }} \sim 10^{-3}$ was measured for $\mathrm{H}_{2} \mathrm{O}$ masers in star-forming regions by Fiebig \& Gusten (1989). In general, the polarization solutions derived here do not apply to $\mathrm{H}_{2} \mathrm{O}$ when more than one hyperfine component is involved. Still, the profile of equation (6.2), displayed in Figure 4 , is a general one and can be expected to describe circular polarization whenever it is generated for $x_{B} \ll 1$. Fiebig \& Gusten analyzed their data with the Troland \& Heiles method, in essence relying only on the shape of this profile. Thus their estimates of magnetic fields of $15-50 \mathrm{mG}$ can be considered reliable. Analysis based on equation (6.3) produces similar results.

The success of theory in explaining all the essential features of maser polarization observations is encouraging. Especially gratifying is the success in explaining the behavior of both circular and linear polarization for different maser transitions of the $\mathrm{SiO}$ molecule. These successes can be considered positive indication that all the relevant ingredients have been properly incorporated into the theory. A major component still missing is Faraday rotation. The study of this effect is left for future work.

I thank R. Barvainis and K. Menten for useful information regarding $\mathrm{SiO}$ and methanol, respectively. This work was supported by NSF grant AST 93-21847. 


\section{APPENDIX A}

\section{RADIATIVE TRANSFER AND ABSORPTION COEFFICIENTS}

General transfer equations for the Stokes parameters of line radiation were derived by Litvak (1975) and summarized in Paper II. They are reproduced here for completeness. For any spin transition, the radiative interactions with photons of polarization $\Delta m$ $(=+1,0,-1)$ are characterized by an absorption coefficient $\kappa^{\Delta m}$. Introduce

$$
\kappa^{1}=\frac{1}{2}\left(\kappa^{+}+\kappa^{-}\right) \text {, }
$$

the mean absorption coefficient for $\Delta m= \pm 1$ transitions, and the three linear combinations

$$
\begin{aligned}
\kappa_{m} & =\frac{1}{2}\left[\kappa^{1}\left(1+\cos ^{2} \theta\right)+\kappa^{0} \sin ^{2} \theta\right], \\
\kappa_{l} & =\frac{1}{2}\left(\kappa^{1}-\kappa^{0}\right) \sin ^{2} \theta, \\
\kappa_{c} & =\frac{1}{2}\left(\kappa^{+}-\kappa^{-}\right) \cos \theta,
\end{aligned}
$$

where $\theta$ is the angle of the radiation propagation direction from the quantization axis. Then the radiative transfer equations are

$$
\begin{aligned}
& \frac{d I}{d l}=\kappa_{m} I+\kappa_{l} Q+\kappa_{c} V, \\
& \frac{d Q}{d l}=\kappa_{m} Q+\kappa_{l} I, \\
& \frac{d U}{d l}=\kappa_{m} U, \\
& \frac{d V}{d l}=\kappa_{m} V+\kappa_{c} I .
\end{aligned}
$$

The basic absorption coefficients $\kappa^{\Delta m}$ are derived from the solution of the steady state level population equations. As a concrete example, consider the standard model of a spin $1 \rightarrow 0$ transition whose three upper levels are denoted $(1, m)($ so that $\Delta m=m)$. The loss rate $\Gamma$ is assumed equal for all states, for simplicity, and the pump rates are $\boldsymbol{P}_{0}$ for the lower level and $\boldsymbol{P}_{1 m}\left(>\boldsymbol{P}_{0}\right)$ for the $(1, m)$ upper level. The pump rates are functions of the particle velocity distribution, assumed Gaussian. To simplify matters further, the geometry is assumed linear so velocities are directly related to frequency shifts from line center and the velocity Gaussian width translates to $\Delta v_{\mathrm{D}}$. Denote by $J_{\Delta m}$ the angle-averaged intensity at the frequency of the $(1, m) \rightarrow 0$ transition and by $B$ the mean $B$-coefficient. Then the steady state populations, $n_{1 m}$ for an upper state and $n_{0}$ for the lower state, are determined from

where

$$
P_{1 m}=\Gamma n_{1 m}+B f_{\Delta m} J_{\Delta m}\left(n_{1 m}-n_{0}\right) \quad m=\Delta m= \pm 1,0, \quad P_{0}=\Gamma n_{0}-B \sum_{p} f_{p} J_{p}\left(n_{1 p}-n_{0}\right)
$$

$$
\begin{array}{ll}
f_{0}=\frac{1}{2}(1-q) \sin ^{2} \theta, & f_{ \pm}=f_{1} \pm w \\
f_{1}=\frac{1}{4}\left(1+\cos ^{2} \theta+q \sin ^{2} \theta\right), & w=\frac{1}{2} v \cos \theta .
\end{array}
$$

The dimensionless factors $f_{\Delta m}$ account for the radiative coupling of different polarizations (see GKK; Papers I, II); note that $f_{+}+f_{-}+f_{0}=2 f_{1}+f_{0}=1$.

The four rate equations provide the three equations for the population differences $n_{1 m}-n_{0}$, which can be rewritten as a set of equations for the three absorption coefficients $\kappa^{\Delta m}$ :

$$
\left(1+f_{\Delta m} J_{\Delta m} / J_{s}\right) \kappa^{\Delta m}+\sum_{\Delta m^{\prime}} f_{\Delta m^{\prime}}\left(J_{\Delta m^{\prime}} / J_{s}\right) \kappa^{\Delta m^{\prime}}=\kappa_{0}^{\Delta m} .
$$

Here $J_{s}=\Gamma / B,{ }^{5}$ and $\kappa_{0}^{\Delta m} \propto\left(P_{1 m}-P_{0}\right) / \Gamma$ are the appropriate unsaturated absorption coefficients, corresponding to the level populations in the limit $J=0$. If we denote by $\sigma$ the sum on the left-hand side, the absorption coefficients are

$$
\kappa^{\Delta m}=\frac{\kappa_{0}^{\Delta m}-\sigma}{1+f_{\Delta m} J_{\Delta m} / J_{s}} .
$$

Inserting these results back into the summation defining $\sigma$ yields

$$
\sigma=\frac{\sum e_{\Delta m} \kappa_{0}^{\Delta m}}{1+\sum e_{\Delta m}}, \quad \text { where } \quad e_{\Delta m}=\frac{f_{\Delta m} J_{\Delta m}}{J_{s}+f_{\Delta m} J_{\Delta m}} .
$$

This completes the definition of $\kappa^{\Delta m}$ in terms of the unsaturated absorption coefficients $\kappa_{0}^{\Delta m}$ and the angle-averaged intensity. The results can also be expressed as rational functions

$$
\kappa^{\Delta m}=N^{\Delta m} / D
$$

${ }^{5}$ In the scalar maser model the definition is $J_{s}=\Gamma /(2 B)$. 
where

$$
\begin{aligned}
D & =1+2\left(j_{0}+j_{+}+j_{-}\right)+3\left(j_{0} j_{+}+j_{+} j_{-}+j_{-} j_{0}\right)+4 j_{0} j_{+} j_{-}, \\
N^{+} & =\left(1+2 j_{0}+2 j_{-}+3 j_{0} j_{-}\right) \kappa_{0}^{+}-j_{-}\left(1+j_{0}\right) \kappa_{0}^{-}-j_{0}\left(1+j_{-}\right) \kappa_{0}^{0}, \\
N^{-} & =-j_{+}\left(1+j_{0}\right) \kappa_{0}^{+}+\left(1+2 j_{0}+2 j_{+}+3 j_{0} j_{+}\right) \kappa_{0}^{-}-j_{0}\left(1+j_{+}\right) \kappa_{0}^{0}, \\
N^{0} & =-j_{+}\left(1+j_{-}\right) \kappa_{0}^{+}-j_{-}\left(1+j_{+}\right) \kappa_{0}^{-}+\left(1+2 j_{+}+2 j_{-}+3 j_{+} j_{-}\right) \kappa_{0}^{0},
\end{aligned}
$$

and where $j_{\Delta m}=f_{\Delta m} J_{\Delta m} / J_{s}$.

The absorption coefficients of a polarized maser resemble those of three scalar masers whose unsaturated absorption coefficients are reduced by $\sigma$, an intensity-dependent common factor reflecting coupling through the shared lower level. When $f_{\Delta m} J_{\Delta m} \ll J_{s}$ (the maser is unsaturated at the frequencies of all three transitions), $e_{\Delta m} \simeq 0$ and $\sigma \simeq 0$ so that $\kappa^{\Delta m} \simeq \kappa_{0}^{\Delta m}$, as should be. In the opposite limit, in which all three transitions are strongly saturated, $e_{\Delta m} \simeq 1$ and $\sigma \simeq \frac{1}{4} \sum \kappa_{0}^{\Delta m}$ so that

$$
\kappa^{\Delta m} \simeq \frac{3 J_{s}}{4 f_{\Delta m} J_{\Delta m}}\left(\kappa_{0}^{\Delta m}-\frac{1}{3} \sum_{\Delta m^{\prime} \neq \Delta m} \kappa_{0}^{\Delta m^{\prime}}\right) .
$$

In order for all three transitions to remain inverted in the limit of strong saturation, the unsaturated absorption coefficient of each transition must exceed the sum of the other two by at least $\frac{1}{3}$. From this it follows that the bounds

$$
\kappa_{0}^{0}<\kappa_{0}^{+}+\kappa_{0}^{-}<3 \kappa_{0}^{0}
$$

must be obeyed whenever all three transitions couple at the same frequency, placing limitations on potential pumping schemes.

\section{APPENDIX B}

\section{ELECTRIC FIELDS AND PHASES}

Maser photons are generated in stimulated emissions. An induced photon has both the frequency and wave vector of the parent photon, but not necessarily its polarization. Indeed, the polarization of the induced photon is determined by the change in magnetic quantum number of the interacting particle, not the polarization of the parent photon. Consider, for example, a photon produced in a $\Delta m=0$ transition, and thus linearly polarized. Because such a photon can also be described as a coherent mixture of two circularly polarized photons, it can induce transitions with any value of $\Delta m$, thus any polarization. For instance, in the case of a spin $1 \rightarrow 0$ induced transition, when the interacting particle is in the $m=0$ state, the induced photon, too, is linearly polarized. But when that particle is in one of the $m= \pm 1$ states, the induced photon is circularly polarized, although the interaction amplitude is reduced.

The polarization solutions correspond to waves with electric fields whose phases are properly tuned to the level populations determined by the pump rates so that induced radiation maintains the polarization of the original one. Methods to determine such electric fields were introduced in Paper I and are easily generalized to the solutions derived here. Two coordinate frames are defined by the problem. One, the $k$-frame, has its $z$ axis aligned with the direction of wave propagation. Components in this frame are denoted by subscripts. The other, the $B$-frame, has its $z$ axis aligned with the quantization axis and components in this frame are denoted by superscripts. The common $y$ axis is perpendicular to the plane that contains the $z$ axes of the two frames. Since the electric field of the wave is a vector, its components in either frame can be obtained from those in the other by a simple transformation. In the case of fully resolved Zeeman patterns, only one $\Delta m$ transition is coupled and only one of the $B$-frame components of the electric field does not vanish at a given frequency. There is no phase relation to be determined in this case and the solution is fully prescribed in Paper I.

When all three components overlap, as is the case for small $x_{B}$ or anisotropic pumping, denote by $E^{\Delta m}$ the proper circular component of the electric field in the $B$-frame. The corresponding intensity $I^{\Delta m} \propto\left|E^{\Delta m}\right|^{2}$ obeys $I^{\Delta m}=f_{\Delta m} I$. Therefore, if we introduce the complex unit vector $\boldsymbol{e}=\boldsymbol{E} /|\boldsymbol{E}|$, its circular components in the $B$-frame are

$$
e^{0}=\sqrt{f_{0}}, \quad e^{+}=\sqrt{f_{+}} e^{i \phi_{+}}, \quad e^{-}=\sqrt{f_{-}} e^{i \phi-},
$$

where $\phi_{ \pm}$are phase angles. The choice of a real phase for $e^{0}$ is always possible because the overall phase of $e$ is irrelevant. With these definitions, the rectangular $k$-frame components of $e$ are

$$
\begin{aligned}
& e_{x}=\frac{1}{\sqrt{2}}\left(\sqrt{f_{+}} e^{i \phi_{+}}+\sqrt{f_{-}} e^{i \phi_{-}}\right) \cos \theta+\sqrt{f_{0}} \sin \theta, \\
& e_{y}=\frac{i}{\sqrt{2}}\left(\sqrt{f_{+}} e^{i \phi_{+}}-\sqrt{f_{-}} e^{i \phi_{-}}\right), \\
& e_{z}=\frac{-1}{\sqrt{2}}\left(\sqrt{f_{+}} e^{i \phi_{+}}+\sqrt{f_{-}} e^{i \phi_{-}}\right) \sin \theta+\sqrt{f_{0}} \cos \theta .
\end{aligned}
$$


Propagating waves are transverse, i.e., $e_{z}=0$, and the normalized Stokes parameters are determined from the two nonvanishing components via $q=e_{y}^{2}-e_{x}^{2}, u=2 \operatorname{Re}\left(e_{x} e_{y}^{*}\right), v=2 \operatorname{Im}\left(e_{x} e_{y}^{*}\right)$. Since the transverse condition $e_{z}=0$ is a complex relation, it provides two equations. From the imaginary part,

$$
-\sqrt{f_{+}} \sin \phi_{+}=\sqrt{f_{-}} \sin \phi_{-} \equiv \sqrt{f_{1}} \sin \phi,
$$

so the field structure is determined by a single phase, $\phi$, whose magnitude is obtained from the real part of the transverse condition:

$$
\cos ^{2} \phi=\frac{f_{0}^{2}+w^{2} \tan ^{4} \theta}{2 f_{0} f_{1} \tan ^{2} \theta}=1-\frac{1-q^{2}-v^{2}}{(1-q)\left(1+\cos ^{2} \theta+q \sin ^{2} \theta\right)} .
$$

This completes the definition of the vector $\boldsymbol{e}$ for a polarization mode characterized by a specific set of $q, u$, and $v$ or, equivalently, $f_{+}$, $f_{-}$, and $f_{0}$. The results are essentially kinematic relations obeyed by all polarization modes. Stationary modes are distinguished as those that maintain a constant unit vector $e$, requiring special phases. Type 1 polarizations have $q^{2}+v^{2}=1$, therefore they have $\phi=0$, except for $q=+1$ which has $\phi= \pm \frac{1}{2} \pi$ (so this is never the $v \rightarrow 0$ limit of another type 1 polarization). In the case of type 0 solutions, the level population equations determine the stationary, $J$-independent part of the structure functions as $f_{0}=$ $\left(3-2 R_{1}\right) /\left(2 R_{1}+1\right), f_{1}=\left(2 R_{1}-1\right) /\left(2 R_{1}+1\right)$. Therefore

$$
\cos ^{2} \phi=\frac{3-2 R_{1}}{2\left(2 R_{1}-1\right) \tan ^{2} \theta}+\frac{8 R_{c}^{2} \tan ^{2} \theta}{\left(3-2 R_{1}\right)\left(2 R_{1}-1\right)}
$$

for all type 0 polarizations, where $R_{1}$ and $R_{c}$ are the ratios appropriate for the particular case. Various bounds and properties of the type 0 solutions for small $x_{B}$ and for antisotropic pumping find simple explanations in terms of the variation of the phase $\phi$ with pump rates and the requirement that it be real (i.e., $\left.0 \leq \cos ^{2} \phi \leq 1\right)$ for physical solutions.

Abraham,Z., \& Vilas Boas, J. W. S. 1994, A\&A, 290, 956

Barvainis, R., \& Deguchi, S. 1989, AJ, 97, 1089

Barvainis, R., McIntosh, G., \& Predmore, C. R. 1987, Nature, 389, 613

Bloemhof, E. E., Reid, M. J., \& Moran, J. M. 1992, ApJ, 397, 500

Claussen, M. J., \& Fix, J. D. 1982, ApJ, 263, 153

Cohen, R. J., et al. 1987, MNRAS, 225, 491

Deguchi, S., \& Watson, W. D. 1986, ApJ, 300, L15

Elitzur, M. 1990, ApJ, 363, 628

. 1991, ApJ, 370, 407 (Paper I)

1993, ApJ, 416, 256 (Paper II)

. 1995, ApJ, 440, 345

Fiebig, D., \& Gusten, R. 1989, A\&A, 214, 333

Gaines, L., Casleton, K., \& Kukolich, S. 1974, ApJ, 191, L99

Garay, G., Moran, J. M., \& Haschick, A. 1989, ApJ, 338, 244

\section{REFERENCES}

Garcia-Barreto, J. A., Burke, B. F., Reid, M. J., Moran, J. M., Haschick, A. D. \& Schilizzi, R. T. 1988, ApJ, 326, 954

Golderich, P., Keeley, D. A., \& Kwan, J. Y. 1973, ApJ, 179, 111 (GKK)

Johnston, I. D. 1967, ApJ, 150, 333

Koo, B., Williams, D. R. W., Heiles, C., \& Backer, D. C. 1988, ApJ, 326, 931

Litvak, M. M. 1975, ApJ, 202, 58

McIntosh, G., \& Predmore, C. R. 1991, in Skyline: Proc. 3rd Haystack Conf., ed. A. D. Haschick \& P. T. P. Ho (ASP Conf. Ser., 16), 83

McIntosh, G., \& Predmore, C. R. 1993, ApJ, 404, L71

McIntosh, G., Predmore, C. R., \& Patel, N. A. 1994, ApJ, 428, L29

Reid, M. J., et al. 1979, ApJ, 227, L89

Troland, T., \& Heiles, C. 1982, ApJ, 252, 179

Zell, P. J., \& Fix, J. D. 1991, ApJ, 369, 506 